\title{
Longitudinal vehicle dynamics control for improved vehicle safety
}

\author{
Herman A. Hamersma*, P. Schalk Els \\ Department of Mechanical and Aeronautical Engineering, University of Pretoria, South Africa
}

\begin{abstract}
The aim is to investigate the improvements in vehicle safety that can be achieved by limiting the vehicle speed based on GPS path information. The control strategy is aimed at reducing vehicle speed before a potentially dangerous situation is reached, in contrast with widely used stability control systems that only react once loss of control by the driver is imminent. An MSC.ADAMS/View simulation model of an off-road test vehicle was developed and validated experimentally. A longitudinal speed control system was developed by generating a reference speed based on the path information. This reference speed was formulated by taking into account the vehicle's limits due to lateral acceleration, combined lateral and longitudinal acceleration and the vehicle's performance capabilities. The model was used to evaluate the performance of the control system on various tracks. The control system was implemented on the test vehicle and the performance was evaluated by conducting field tests. Results of the field tests indicated that the control system limited the acceleration vector of the vehicle's centre of gravity to prescribed limits, as predicted by the simulations, thereby decreasing the possibility of accidents caused by rollover or loss of directional control due to entering curves at inappropriately high speeds.
\end{abstract}

Keywords: Autonomous vehicles; Vehicle safety; Vehicle modelling; Path planning; Off-road vehicles

\section{Introduction}

Applications of automation in vehicle engineering range from rain sensing windscreen wipers to climate control systems. More specific to the study of vehicle dynamics is the improvement achievable by implementing feedback control systems which influence the dynamic behaviour of the vehicle with regards to the six degrees of freedom, namely lateral, vertical and longitudinal translation as well as roll, pitch and yaw rotation. Application of automation to control these degrees of freedom may lead to the optimisation of vehicle utilisation.

\footnotetext{
* Corresponding author.

E-mail addresses: hhamersma@gmail.com (H.A. Hamersma), schalk. els@up.ac.za (P.S. Els).
}

\subsection{Fully autonomous vehicles}

One of the best examples of the application of automation in the modern engineering fraternity was during the 2005 DARPA Grand Challenge [1] and the 2007 DARPA Urban Challenge [2]. Both these Challenges required vehicles to negotiate terrains that represent everyday driving conditions (especially from a military point of view) and hence path planning played an important role in successfully completing these Challenges. The 2005 DARPA Grand Challenge was won by Stanford University's 'Stanley' [1] and the 2007 DARPA Urban Challenge was won by 'Boss', the entry from Carnegie Mellon University, General Motors, Caterpillar, Continental and Intel [2]. 'Stanley' managed an average speed of $30.7 \mathrm{~km} / \mathrm{h}$ [1] and 'Boss' an average speed of $22.5 \mathrm{~km} / \mathrm{h}$ [2]. Due to the fairly low speeds involved, most DARPA Challenge entries employed simple 


\section{Nomenclature}

\begin{tabular}{|c|c|c|c|}
\hline Symbol & Description & $R$ & radius of curve $(\mathrm{m})$ \\
\hline$A_{\text {Brakes }}$ & acceleration due to braking $\left(\mathrm{m} / \mathrm{s}^{2}\right)$ & $\Delta s_{i}$ & length of $i$ th track segment $(\mathrm{m})$ \\
\hline & projected frontal area $\left(\mathrm{m}^{2}\right)$ & $t$ & time $(\mathrm{s})$ \\
\hline$A_{\text {maxlong }}$ & $\begin{array}{l}\operatorname{maximum} \text { allowed longitudinal acceleration } \\
\left(\mathrm{m} / \mathrm{s}^{2}\right)\end{array}$ & $\begin{array}{l}T \\
T_{E B T}\end{array}$ & $\begin{array}{l}\text { throttle position }(\%) \\
\text { engine brake torque }(\mathrm{N} \mathrm{m})\end{array}$ \\
\hline$A_{x}$ & $\begin{array}{l}\text { acceleration in the } x \text {-direction (longitudinal) } \\
\left(\mathrm{m} / \mathrm{s}^{2}\right)\end{array}$ & $\begin{array}{l}T_{\text {Engine }} \\
t_{w}\end{array}$ & $\begin{array}{l}\text { engine torque }(\mathrm{N} \mathrm{m}) \\
\text { track width }(\mathrm{m})\end{array}$ \\
\hline$A_{y}$ & acceleration in the $y$-direction (lateral) $\left(\mathrm{m} / \mathrm{s}^{2}\right)$ & $u$ & PID controller output (Dimensionless) \\
\hline$B_{\kappa}$ & $\begin{array}{l}\text { linear coefficient matrix of quadratic cost func- } \\
\text { tion for minimum curvature formulation } \\
\text { (Dimensionless) }\end{array}$ & $\begin{array}{l}V \\
V_{\text {ref }} \\
x\end{array}$ & $\begin{array}{l}\text { vehicle speed }(\mathrm{m} / \mathrm{s}) \\
\text { reference speed }(\mathrm{m} / \mathrm{s}) \\
\text { coordinate } x \text { value }(\mathrm{m})\end{array}$ \\
\hline$C_{D}$ & coefficient of aerodynamic drag (Dimensionless) & $x_{l}$ & left edge (bound) of road's $x$-coordinate (m) \\
\hline$c_{i}$ & $\begin{array}{l}\text { constant term of straight line describing perpen- } \\
\text { dicular bisector }(\mathrm{m})\end{array}$ & $x_{q}, i$ & $\begin{array}{l}X \text {-coordinate of centre point of chord of } i \text { th } \\
\text { segment of trajectory }(\mathrm{m})\end{array}$ \\
\hline$d_{\text {prev }}$ & preview distance $(\mathrm{m})$ & $x_{r}$ & right edge (bound) of road's $x$-coordinate $(\mathrm{m})$ \\
\hline$e^{p}$ & velocity error $(\mathrm{m} / \mathrm{s})$ & $x_{R, i}$ & $X$-coordinate of centre point of arc of $i$ th \\
\hline$F_{D}$ & demand force $(\mathrm{N})$ & & segment of trajectory $(\mathrm{m})$ \\
\hline$F_{\text {Drag }}$ & Force due to aerodynamic drag $(\mathrm{N})$ & $\Delta X_{i}$ & change in $x$-distance of $i$ th track segment (m) \\
\hline$F_{r r}$ & force due to rolling resistance $(\mathrm{N})$ & $y$ & coordinate $y$ value $(\mathrm{m})$ \\
\hline$F_{\text {incl }}$ & force due to longitudinal road inclination $(\mathrm{N})$ & $y_{l}$ & left edge (bound) of road's $y$-coordinate $(\mathrm{m})$ \\
\hline & $\begin{array}{l}\text { gravitational acceleration }\left(\mathrm{m} / \mathrm{s}^{2}\right) \\
\text { centre of gravity height }(\mathrm{m})\end{array}$ & $y_{q}, i$ & $\begin{array}{l}Y \text {-coordinate of centre point of chord of } i \text { th } \\
\text { segment of trajectory }(\mathrm{m})\end{array}$ \\
\hline$H_{\kappa}$ & $\begin{array}{l}\text { hessian matrix of quadratic cost function for } \\
\text { minimum curvature formulation (Dimension- } \\
\text { less) }\end{array}$ & $\begin{array}{l}y_{r} \\
y_{R, i},\end{array}$ & $\begin{array}{l}\text { right edge (bound) of road's } y \text {-coordinate }(\mathrm{m}) \\
Y \text {-coordinate of centre point of arc of } i \text { th } \\
\text { segment of trajectory }(\mathrm{m})\end{array}$ \\
\hline$\hat{i}$ & unit vector in $x$-direction (Dimensionless) & $\Delta y_{i}$ & change in $y$-distance of $i$ th track segment (m) \\
\hline & unit vector in $y$-direction (Dimensionless) & $\alpha$ & parameter identifying position of vehicle on the \\
\hline$K_{D}$ & PID derivative gain (Dimensionless) & & road (Dimensionless) \\
\hline$K_{I}$ & PID integral gain (Dimensionless) & $\alpha_{\kappa}$ & parameter identifying position of vehicle on the \\
\hline $\begin{array}{l}K_{P} \\
M\end{array}$ & $\begin{array}{l}\text { PID proportional gain (Dimensionless) } \\
\text { vehicle mass }(\mathrm{kg})\end{array}$ & & $\begin{array}{l}\text { road for minimum curvature formulation } \\
\text { (Dimensionless) }\end{array}$ \\
\hline \multirow[t]{2}{*}{$m_{i}$} & gradient of chord of $i$ th segment of & $\theta$ & inclination angle (radians) \\
\hline & (Dim & $\kappa$ & ectory (Dimensionless) \\
\hline$m_{i}^{\prime}$ & $\begin{array}{l}\text { gradient of perpendicular bisector of chord of } \\
i \text { th segment of trajectory (Dimensionless) }\end{array}$ & $\begin{array}{l}\mu \\
\mu_{r r}\end{array}$ & $\begin{array}{l}\text { tyre-road friction coefficient (Dimensionless) } \\
\text { coefficient of rolling resistance (Dimensionless }\end{array}$ \\
\hline$n$ & & $\begin{array}{l}\mu_{r r} \\
\rho\end{array}$ & density of air $\left(\mathrm{kg} / \mathrm{m}^{3}\right)$ \\
\hline$P$ & position of vehicle on track (m) & $\tau$ & preview time $(\mathrm{s})$ \\
\hline$P_{\text {hyd }}$ & hydraulic brake line pressure (MPa) & & \\
\hline & coefficients of 2D polynomial (Dimen & & \\
\hline
\end{tabular}

linear vehicle models to control speed and steering. A possible area of improvement is thus to increase the speed these vehicles attained while competing in the various DARPA Challenges.

\subsection{Driver assist systems}

While the DARPA Challenges specifically aimed at developing fully autonomous vehicles (vehicles that drive with no human input), a more practical and feasible approach would be to develop a control system that can be used as a driver aid. By using sensor technology similar to that employed in the DARPA Challenges (such as numerous LIDARs, Differential GPS, radar and cameras), the vehicle can obtain preview information of its immediate surroundings that enables it to identify a suitable path to be followed. This is often referred to as path planning. This path information can subsequently be used for path following where decisions can be made that improve the vehicle's safety. Path planning using technology such as cameras, radar and LIDAR. has been extensively studied. This technology is well commercialised and many vehicles are now fitted with adaptive cruise control, traffic sign recognition, lane departure warning and satellite navigation. All these technologies rely on camera, GPS and radar sensors. In the present study, this is not the contribution to be made. 
The emphasis rather falls on developing a control system that uses information available from these sensors to enhance vehicle safety.

Many off-road vehicles are also used on roads. Due to their high $\mathrm{CG}$ and suspension characteristics being optimised for off-road use, the handling and rollover propensity of these vehicles cause safety concerns when driven at higher speeds on roads. Several commercially available technologies exist to help control the vehicle under impending accident conditions. These include anti-lock brake systems (ABS), electronic stability control (ESC), electronic brake distribution (EBD) and active steering. These systems however only start acting under extreme conditions where the driver and vehicle may already be in trouble. Path following algorithms that take vehicle dynamics and path preview information into account may prevent the vehicle entering a situation where the handling can become unsafe, i.e. before ABS or ESC becomes active. The proposed system thus acts proactive rather than reactive. In many high-end vehicles all the required path preview information is already available as are the actuation systems for steering and braking. The proposed control system therefore does not require any additional hardware - it is simply a new software algorithm that improves a vehicle's safety. Table 1 shows a list of 'Commercially off the shelf' (COTS) sensors, algorithms and interference techniques available. The focus of this research falls in the column titled 'Algorithms'.

\subsection{Research question and focus of current paper}

The research question of this paper may thus be defined as: to develop a longitudinal control system for path planning and following that takes into account the nonlinear vehicle dynamics and uses GPS information for path preview. This control system should intelligently limit the vehicle's speed to prevent the vehicle from exceeding the limits imposed by the vehicle dynamics (i.e. rollover, sliding or overshooting the path) and hence improve the safety of the occupants. The control system is based on trajectory planning algorithm developed by Braghin et al. [3]. Braghin et al. [3] developed a similar control system and evaluated

Table 1

List of COTS sensors, algorithms and interference techniques.

\begin{tabular}{lll}
\hline $\begin{array}{l}\text { Path information } \\
\text { available }\end{array}$ & Algorithms & $\begin{array}{l}\text { Interference techniques } \\
\text { available }\end{array}$ \\
\hline $\begin{array}{l}\text { GPS or satellite } \\
\text { navigation } \\
\text { Vehicle speed }\end{array}$ & $\begin{array}{l}\text { Adaptive cruise } \\
\text { control } \\
\text { Lane departure } \\
\text { warning }\end{array}$ & Electronic power steering \\
Blind spot warning & ABS \\
Radar & $\begin{array}{l}\text { High speed path } \\
\text { following }\end{array}$ & EBD \\
Cameras & & ESC \\
& & $\begin{array}{l}\text { Active or semi-active } \\
\text { suspension control }\end{array}$ \\
& & Torque vectoring \\
\hline
\end{tabular}

its performance with a simplified dynamic model. The results reported by [3] indicated that developing a control system based on optimising the path of a vehicle may result in improved lap times for race drivers. The same approach is followed in this paper but now with the focus on reducing lateral acceleration of the vehicle and hence improving vehicle safety rather than decreasing lap time around a racetrack.

A 1997 Land Rover Defender 300Tdi 110 Wagon is the experimental platform used for this study. Feedback control systems, that can mimic the inputs given by a human driver, have been implemented in such a way that the test vehicle may be driven manually or via a computer. Several driver assist systems that lie somewhere between no control and fully autonomous control, such as an Anti-Lock Braking System (ABS) and Traction Control, can be implemented on the Land Rover test vehicle. The systems implemented on the vehicle consist of:

- A path following steering control system capable of fully autonomous control up to high acceleration limits.

- A braking control system.

- A gear-shift and clutch control system.

- A throttle pedal control system.

A model-based design approach is followed to develop the longitudinal control system and an experimentally validated model is thus required. An existing MSC.ADAMS/ View multi-body dynamics model that accurately captures the fully nonlinear vertical and lateral dynamics of the test vehicle has been developed by Uys et al. [4]. The model is now expanded and experimentally validated to accurately capture the longitudinal dynamics.

The driver models developed by Botha [5] have been updated and improved to provide for a trajectory planning algorithm, based on the work done by Braghin et al. [3]. The longitudinal control system is developed in simulation and finally validated experimentally.

\section{ADAMS model development and validation}

The acceleration and braking performance, aerodynamic drag and rolling resistance of the Land Rover test vehicle had to be characterised experimentally before inclusion in the existing MSC.ADAMS/View model of the Land Rover.

\subsection{Acceleration performance}

The torque delivered by the engine is a function of two parameters, namely engine speed and throttle position. To determine the torque delivered by the engine, strain gauges were applied to both forward and rearward drive shafts in a full-bridge configuration. The strain gauges were calibrated in the laboratory for direct torque measurement by applying known torque values. The torque applied at a constant 
throttle position as maintained by the throttle pedal actuator was measured.

Characterisation of the gearbox, transfer case and differential ratios along with the wheel rolling radius was thus necessary to determine the engine torque, since torque was measured on the drive shafts. By measuring the engine speed and drive shaft speed for various gear selections, the gear ratios and transfer case ratios were determined.

Similarly, by comparing the wheel speed and drive shaft speed, the differential ratio was determined. The rolling radius was measured as the distance from the centre of the wheel to the road on a flat road under static wheel load and a value of $0.386 \mathrm{~m}$ was obtained. Table 2 summarizes these measurements (note that the gear ratios include the transfer case ratio).

Tests were conducted by accelerating in a pre-selected gear from idle speed until a constant vehicle speed was reached on a smooth and level concrete road (the Gerotek Long Straight Track [6]). The throttle position was varied from $25 \%$ of the range of motion, to $100 \%$ (full throttle). The measured torque was then divided by the applicable gear ratio to find the engine torque, assuming $100 \%$ efficiency, i.e. the resulting torque compensates for inefficiencies in the gearbox and transfer case. The driveshaft speed was measured with a proximity switch and a frequency to voltage converter and multiplied by the

Table 2

Gear and differential ratios.

\begin{tabular}{ll}
\hline Variable & Ratio \\
\hline First gear & $5.158: 1$ \\
Second gear & $2.764: 1$ \\
Third gear & $1.737: 1$ \\
Fourth gear & $1.202: 1$ \\
Fifth gear & $0.888: 1$ \\
Differential & $3.45: 1$ \\
\hline
\end{tabular}

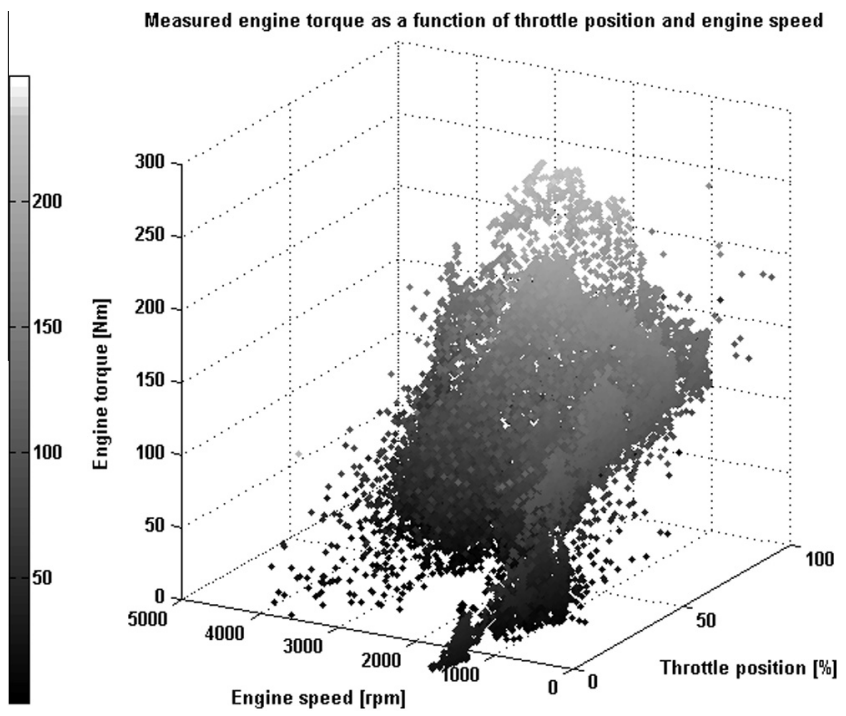

Fig. 1. Measured engine torque as a function of engine speed and throttle position. applicable gear ratio to find the engine speed (although direct measurement of the engine speed is possible). The tests were repeated for various throttle positions, starting from idle speed until a constant speed was reached in various gears. The resulting torque is shown as a function of engine speed and throttle position in Fig. 1.

MATLAB's Surface Fit Toolbox was used to fit a polynomial function to the measured torque. The resulting fit had a $R^{2}$ value of 0.93 and is shown in Fig. 2. The resultant polynomial fit is given by Eq. (1).

$$
\begin{aligned}
T_{\text {Engine }}= & p_{00}+p_{10} T+p_{01} n+p_{20} T^{2}+p_{11} T n+p_{02} T^{2} \\
& +p_{30} T^{3}+p_{21} T^{2} n+p_{12} T^{2}+p_{03} n^{3}+p_{40} T^{4} \\
& +p_{31} T^{3} n+p_{22} T^{2} n^{2}+p_{13} T n^{3}+p_{04} n^{4}
\end{aligned}
$$

with

$$
\begin{array}{ll}
p_{12}=8.905 \\
p_{00}=62.52 & p_{21}=-30.6 \\
p_{10}=83.71 & p_{03}=14.67 \\
p_{01}=-64.18 & p_{40}=1.834 \\
p_{20}=7.657 & p_{31}=3.599 \\
p_{11}=-32.87 & p_{22}=-11.74 \\
p_{02}=-8.014 & p_{13}=9.552 \\
p_{30}=-3.583 & p_{04}=1.366
\end{array}
$$

\subsection{Aerodynamic drag and rolling resistance}

Aerodynamic drag, rolling resistance and the gravity component due to inclination are the only resistive forces acting on the vehicle [7]. These forces are governed by Eq. (2).

$$
\begin{aligned}
F_{D} & =-F_{\text {Drag }}-F_{r r}-F_{\text {Incl }} \\
& =-1 / 2 \rho V^{2} C_{D} A_{f}-\mu_{r r} M g \cos \theta-M g \sin \theta
\end{aligned}
$$

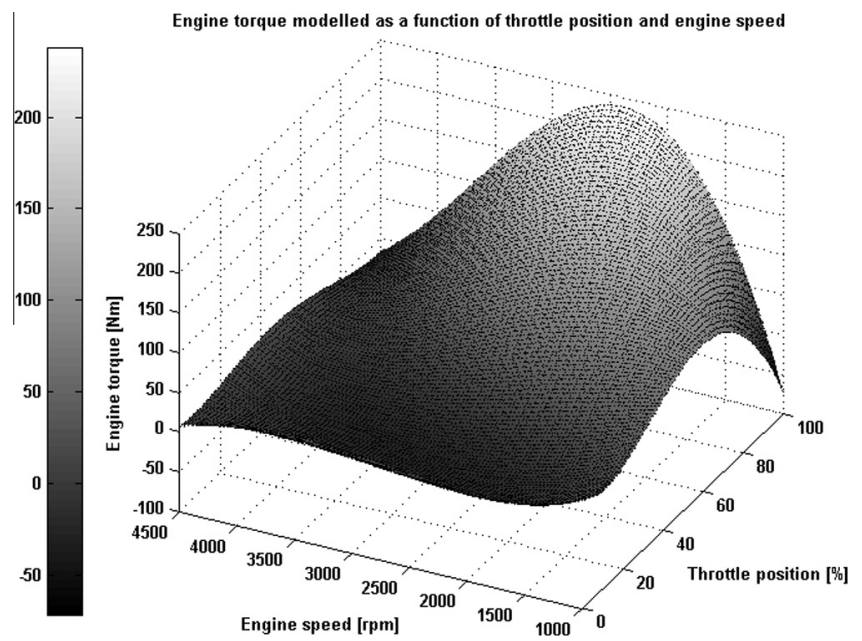

Fig. 2. Modelled engine torque as a function of engine speed and throttle position. 
The resistive forces due to drag and rolling resistance were determined by accelerating the vehicle to $100 \mathrm{~km} / \mathrm{h}$ and then coasting with the manual gearbox in neutral to a standstill on a level road while measuring the vehicle speed. Fig. 3(a) presents the vehicle speed as a function of time. The vehicle speed is differentiated to find acceleration and plotted in Fig. 3(b) as a function of time. Applying Newton's Second Law the resistive force is calculated from the acceleration. The demand force is shown as a function of vehicle speed in Fig. 4.

As may be seen from Eq. (2), the demand forces due to drag and rolling resistance (which in this case includes drivetrain drag and other losses) are a function of vehicle speed squared and a constant. A fit of this form was made through the captured data, resulting in the following coefficients (see Fig. 4):

$\rho C_{D} A_{f}=2.583$

$\mu_{r r}=0.024$

\subsection{Braking}

In addition to the demand forces acting on the vehicle, the vehicle speed can be reduced by applying the vehicle's hydraulically actuated friction brakes or by engine braking. The friction brakes were characterised by accelerating the vehicle to $70 \mathrm{~km} / \mathrm{h}$ and then braking to standstill while measuring the longitudinal acceleration and the brake line hydraulic pressure. Fig. 5 shows that a linear relationship (given by Eq. (5)) exists between brake line pressure and deceleration. Multiplying the deceleration with the vehicle mass gives the braking force.

$A_{\text {Brakes }}=0.832 P_{\text {hyd }}-0.5507$

The engine braking torque is applied to the power train when the driver removes his/her foot from the throttle pedal while the vehicle is moving and in gear. This is due
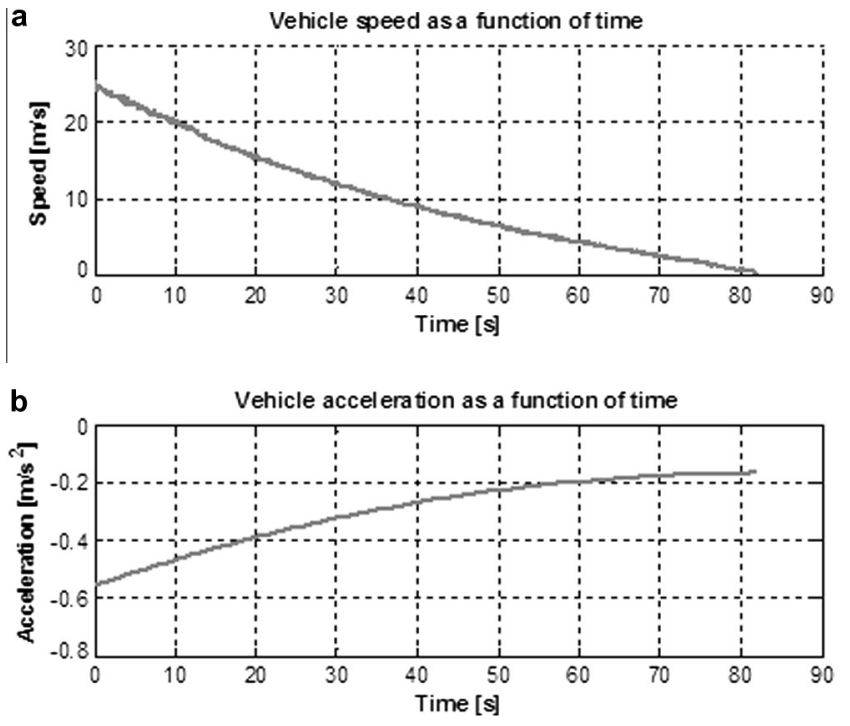

Fig. 3. Coast down experimental results.

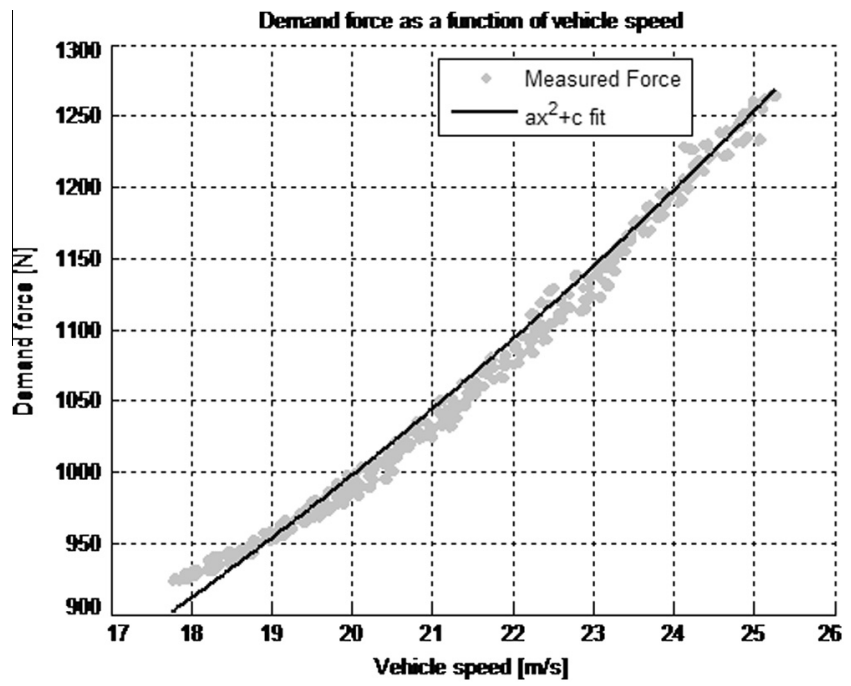

Fig. 4. Demand force due to drag and rolling resistance as a function of vehicle speed.

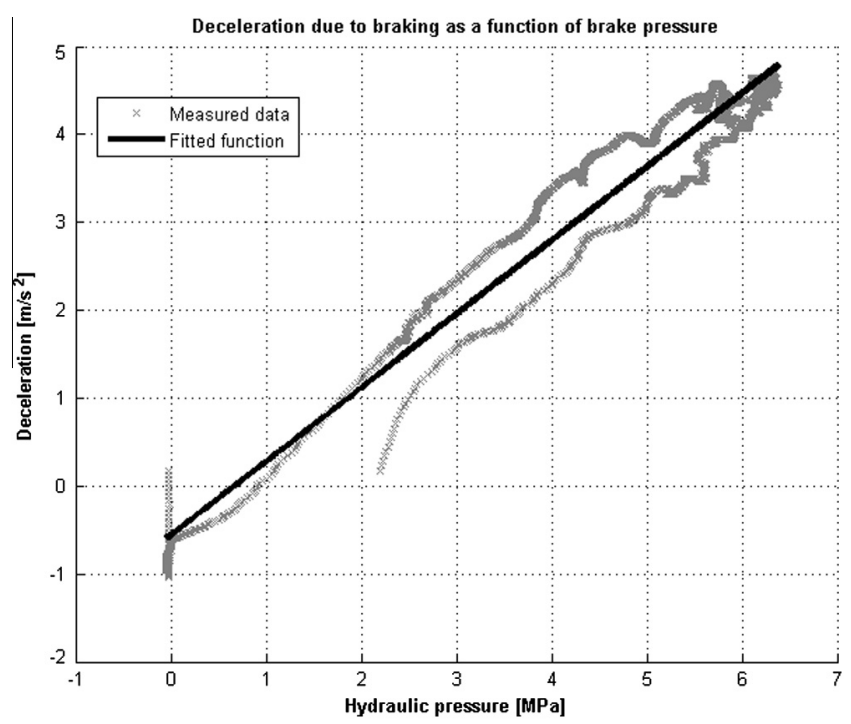

Fig. 5. Longitudinal deceleration as a function of brake line hydraulic pressure.

to the torque required to turn the engine while compressing the air inside the cylinders. This torque is multiplied by all the gear, transfer case and differential ratios that form part of the vehicle's power train and is applied to the driving wheels. Characterisation of the engine braking torque was done by Botha [8]. The measured characteristic is given in Fig. 6 and the governing equation is given by Eq. (6).

$T_{E B T}=3.8713 \times 10^{-6} n^{2}-25.11 \times 10^{-3} n+5.44$

\subsection{Model validation}

The relationships developed in Sections 2.1-2.3 had to be incorporated in the MSC.ADAMS/View model by using co-simulation with MATLAB/Simulink. Fig. 7 


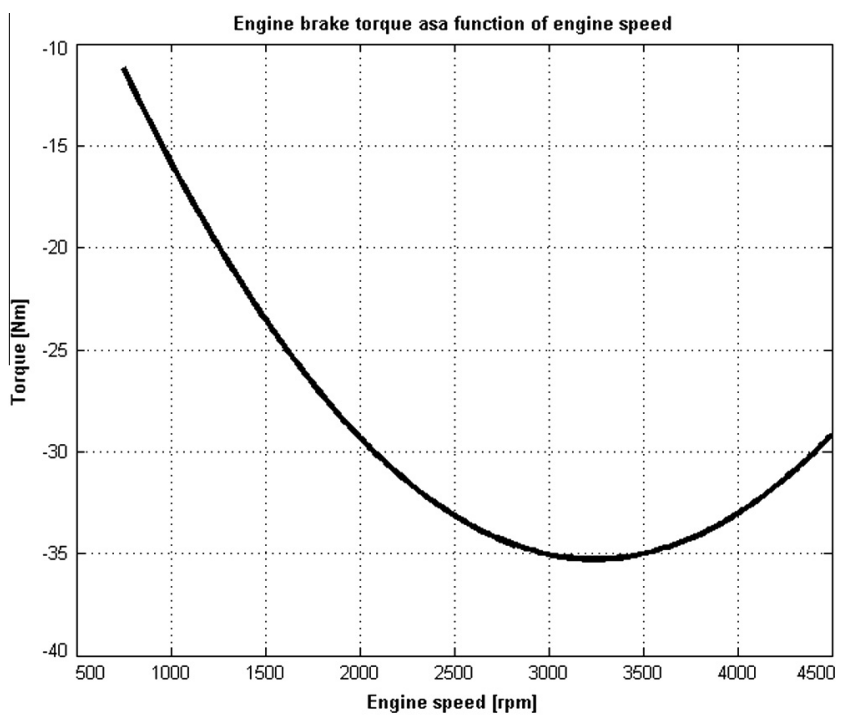

Fig. 6. Engine braking torque as a function of engine speed.

shows a block diagram of the longitudinal model. The variables calculated by the simulation model are vehicle speed and driveshaft speed. The control inputs given are the throttle position, brake line pressure and the gear selected. These measured variables and control inputs are used to determine the driveshaft torque (i.e. supply force) and demand force acting on the vehicle.

A validation run was done with the Land Rover Defender while recording the vehicle's speed, throttle pedal position, brake line hydraulic pressure and clutch pedal position. The validation run consisted of four stages as indicated in Fig. 8:

Stage I. Accelerating from a standing start in first, second and third gear to $60 \mathrm{~km} / \mathrm{h}$ (from 0 to approximately $15 \mathrm{~s}$ ).

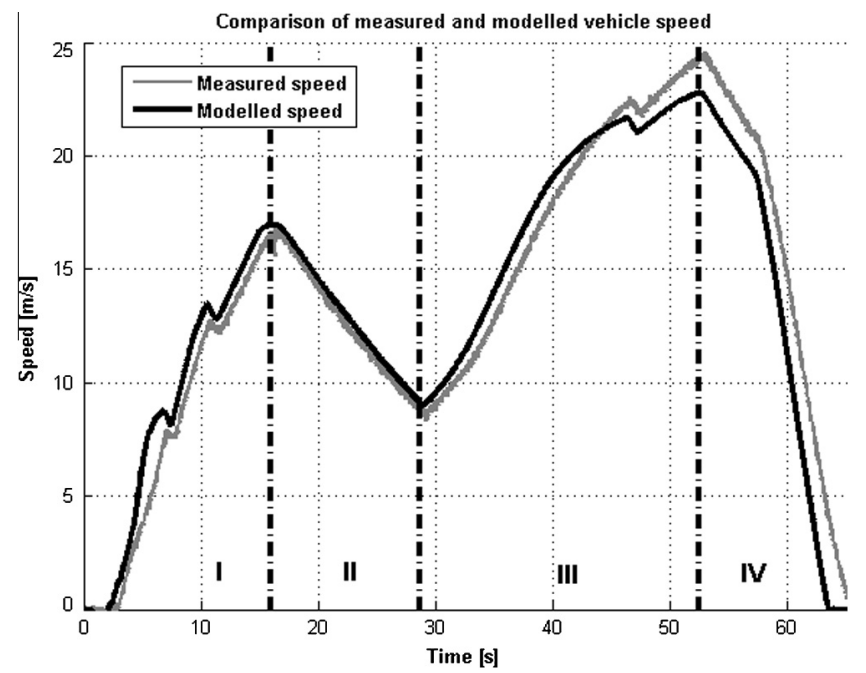

Fig. 8. Comparison of measured and modelled vehicle speeds for validation run.

Stage II. Decelerating with engine braking in third gear from $60 \mathrm{~km} / \mathrm{h}$ back to idle speed (from approximately $15 \mathrm{~s}$ to just before $30 \mathrm{~s}$ ).

Stage III. Accelerating in third and fourth gear from third gear idle speed up to $90 \mathrm{~km} / \mathrm{h}$ (from $30 \mathrm{~s}$ to approximately $55 \mathrm{~s}$ ).

Stage IV. Braking with the clutch disengaged (from $55 \mathrm{~s}$ onwards).

The measured throttle pedal position and brake line pressure were used as inputs to the mathematical model. The gear change timing was accomplished by manually selecting the time (using MATLAB's 'ginput' function) at which the clutch pedal was depressed and incrementing the gear selection at each point.

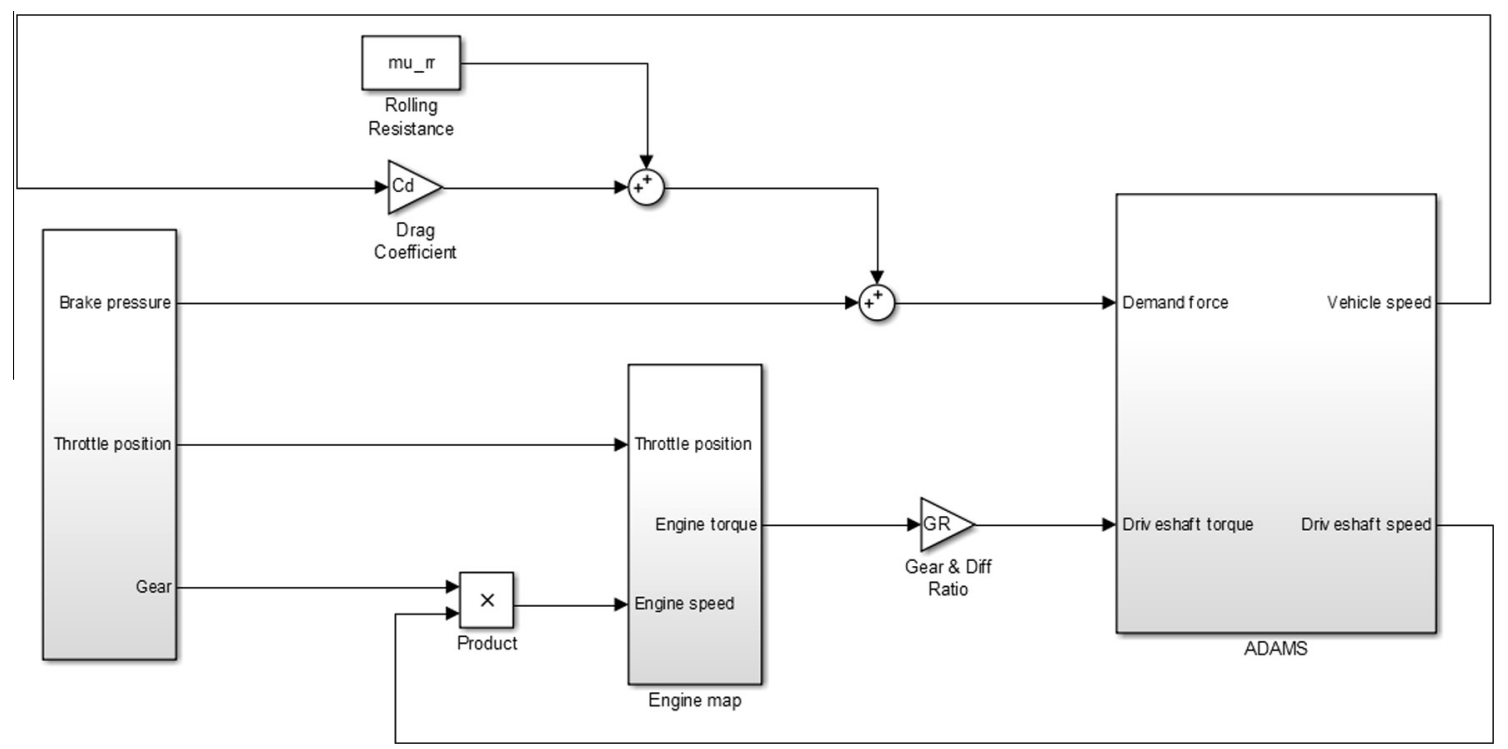

Fig. 7. Schematic layout of mathematical longitudinal model of the Land Rover. 
The resulting speed, as calculated by the model is compared with the measured speed in Fig. 8. Fig. 9 shows the torque applied to the driveshaft during the validation run. As may be seen from Fig. 8 there are some discrepancies present when comparing the modelled speed and measured speed of the vehicle. These errors may be attributed to:

(1) Inaccurate gear change timing.

(2) The validation run was conducted on a slight downhill and the model does not provide for the effect of the component of gravity along an incline (although Eq. (2) provides for the force component due to longitudinal inclination but it was not implemented in the model).

(3) There may have been external contributing factors (such as wind loading) that were not measured and not accounted for in the model.

However, despite these discrepancies, a very close correlation may be seen for both vehicle speed and driveshaft torque in Figs. 8 and 9. Although several improvements and refinements are possible, the model is deemed validated and accurate enough for the current investigation.

\section{Control system development}

A common problem with sports-utility-vehicles is the low rollover threshold, due to a high centre of gravity. The quasi-static rollover threshold is given by Eq. (7) [7]:

$\frac{A_{y}}{g}=\frac{t_{w}}{2 h}$

Similarly, for a simple vehicle model, the vehicle will start sliding when $A_{y} \geqslant \mu g$ [7]. The lateral acceleration experienced by a vehicle when negotiating a corner is given by Eq. (8) [7]. Eq. (8) is derived for steady-state cornering (where the vehicle is traveling at a constant speed and

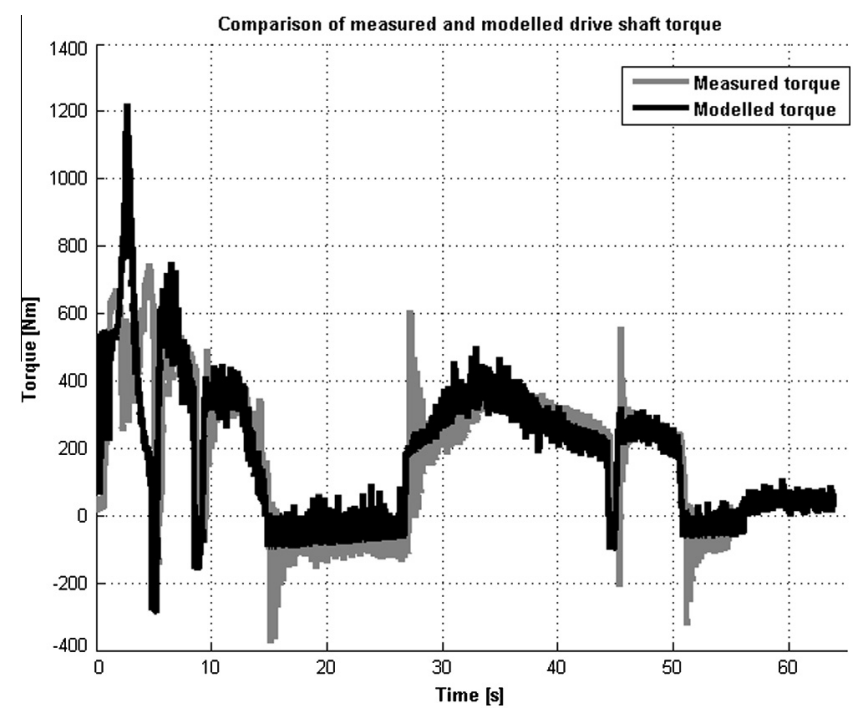

Fig. 9. Measured and modelled driveshaft torque for the validation run. constant yaw rate). The method of reducing the vehicle's rollover propensity investigated by this project is to reduce the lateral acceleration experienced by the vehicle by either by reducing the speed or increasing the turn radius, rather than changing the rollover threshold.

$A_{y}=\frac{V^{2}}{R}$

From Eq. (8) it may be seen that by increasing the radius of the corner being negotiated, the lateral acceleration will be reduced and the speed at which rollover will occur is correspondingly increased, hence the development of a trajectory planning algorithm that maximises the radius of curvature. Although Eq. (8) is developed for steady-state cornering - which may not necessarily be the case during driving - it was assumed that the use of Eq. (8) is sufficient for the purpose of this investigation. The use of an optimisation algorithm to determine the minimum curvature may also result in a "smoother" route, reducing the effect of transient cornering.

The lateral acceleration limit at which sliding or rollover will occur depends on the terrain. While determining a suitable acceleration limit as a function of the terrain is outside the scope of this study, there are several methods that can be used, such as:

- Friction coefficient estimation through parameter estimation.

- Using side slope information (this may significantly reduce the lateral acceleration at which rollover or sliding may occur).

- Driver input by selecting the type of terrain (e.g. sand or mud) or driver aid input (through a terrain response algorithm).

The idea is thus to, based on information received from sensors such as GPS and radar, determine a suitable vehicle speed to keep the longitudinal and lateral acceleration of the vehicle below prescribed limits to keep the vehicle safe. This reduction in speed must be sufficient to prevent electronic stability control from being activated. The focus is also not on path planning but rather on the vehicle dynamics required to follow a path safely.

\subsection{Trajectory planning using minimum curvature approach}

The roads or tracks used for simulation and experimental purposes are defined using GPS coordinates (latitude and longitude), which can be converted to $x$ and $y$ coordinates of the road centreline. By specifying the road width, the road boundaries may also be determined. Trajectory planning is concerned with determining the path the vehicle must follow when negotiating a specified road or track. The trajectory may be optimised for various operating conditions. Braghin et al. [3] developed two such methods - optimising for the shortest route and optimising for the minimum curvature. The minimum curvature formulation is applicable for the 
case under investigation, wherein minimising the curvature of the path followed by the vehicle will result in maximizing the radius of the trajectory and hence lowering the lateral acceleration (see Eq. (8)).

The approaches followed by Braghin et al. [3] rely on discretizing the road into segments. Given the road's centreline coordinates, the left and right edges (or boundaries) of the road is then determined by assuming a track width (or using the actual measured track width). The position of the vehicle on the road (which must lie within the bounds of the road) is then given by Eq. (9), where the position depends on the variable $\alpha$ (which is allowed to vary between zero and unity, zero resulting in the position being on the right boundary and unity on the left boundary) [3]:

$P=x_{r}+\alpha\left(x_{l}-x_{r}\right) \hat{i}+y_{r}+\alpha\left(y_{l}-y_{r}\right) \hat{j}$

Braghin et al. [3] then formulates a bound quadratic optimisation problem which, depending on the formulation, results in the shortest distance or minimum curvature trajectory, with $\alpha$ being the independent variable to be optimised. The cost function of the quadratic optimisation problem for the shortest distance trajectory is formulated from the distance formula in Cartesian coordinates and is given by Eq. (10).

$\kappa^{2}=\frac{1}{2}\left\langle\alpha_{\kappa}\right\rangle\left[H_{\kappa}\right]\left\{\alpha_{\kappa}\right\}+B_{\kappa}\left\{\alpha_{\kappa}\right\}$

Using MATLAB's quadratic optimisation function, the trajectory's curvature is minimised. Fig. 10 shows the trajectory as optimised for Gerotek's ride and handling track [6].

Systems such as satellite navigation and lane departure warning system are readily available in the commercial vehicle market. Driver assist systems such as these may be used to augment the trajectory planning methods proposed by Braghin et al. [3]. A commercial GPS (with a typical accuracy of $15 \mathrm{~m}$ that updates its position approximately once per second [9] may be used in conjunction with a satellite navigation system, such as described by Dork [10], to determine the vehicle's whereabouts on a map and what lies ahead, in essence determine the centreline of the road or track the vehicle is negotiating. Lane Departure Warning Systems, such as developed by Batavia et al. [11], are now commercially available and may be used to detect the road boundaries and the vehicle's position relative to the road boundaries. With knowledge of the road centreline from the satellite navigation system and the vehicle's position relative to the road boundaries, vehicle to vehicle communication $(\mathrm{V} 2 \mathrm{~V})$ or radar information, trajectory planning may readily be implemented in commercial vehicles using existing sensors.

\subsection{Speed profile}

Once the minimum curvature trajectory has been determined, a reference speed at which the vehicle will attempt

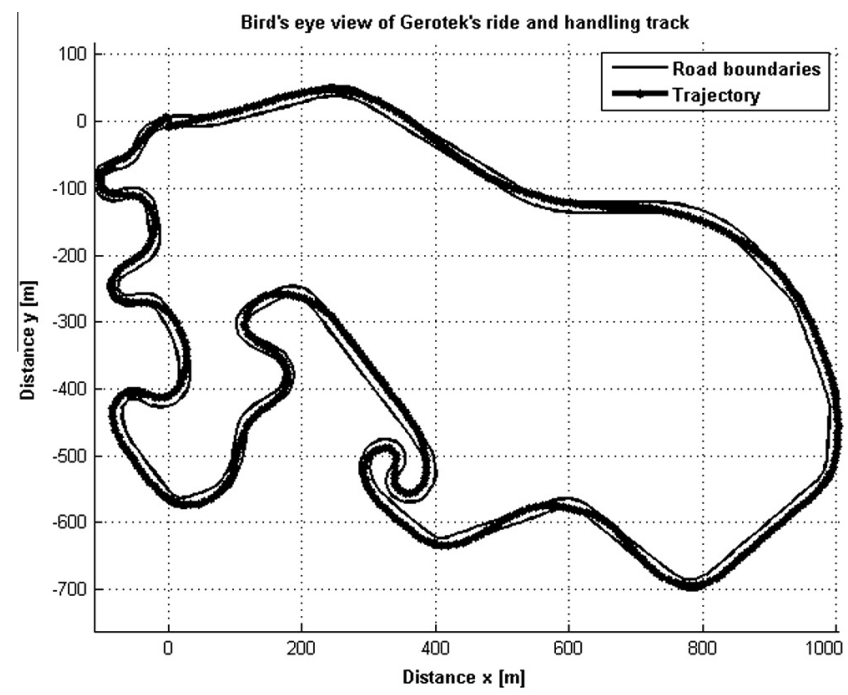

Fig. 10. Minimised curvature trajectory of Geroteks ride and handling track [6].

to negotiate the track must be determined. This speed is limited by three factors, namely:

- Lateral acceleration limit.

- Tyre contact force limit when longitudinal and lateral acceleration is present.

- The vehicle's limitations with regards to longitudinal acceleration and deceleration due to the engine and/or braking system.

The process followed to formulate the speed profile is given schematically in Fig. 11.

\subsubsection{Speed limit due to lateral acceleration}

As is evident from Eq. (8), the speed limit due to lateral acceleration is a function of the radius of the trajectory being followed. An algorithm to determine the radius of the trajectory for each segment of the trajectory was developed. Given three points, it is always possible to draw an arc with a constant radius that passes through all three points. The perpendicular bisectors of the chords joining adjacent points pass through the centre of the arc. The distance from the centre of the arc to any of the three points used is the radius of curvature. Fig. 12 shows the result of this procedure.

The mathematical operations needed to determine the radius of curvature following this procedure will now be explained. First, the perpendicular bisectors are determined. The bisectors are located at the centre of each chord, thus the centre points of each chord have to be determined:

$x_{q, i}=\frac{1}{2}\left(x_{i}+x_{i+1}\right)$

$y_{q, i}=\frac{1}{2}\left(y_{i}+y_{i+1}\right)$ 


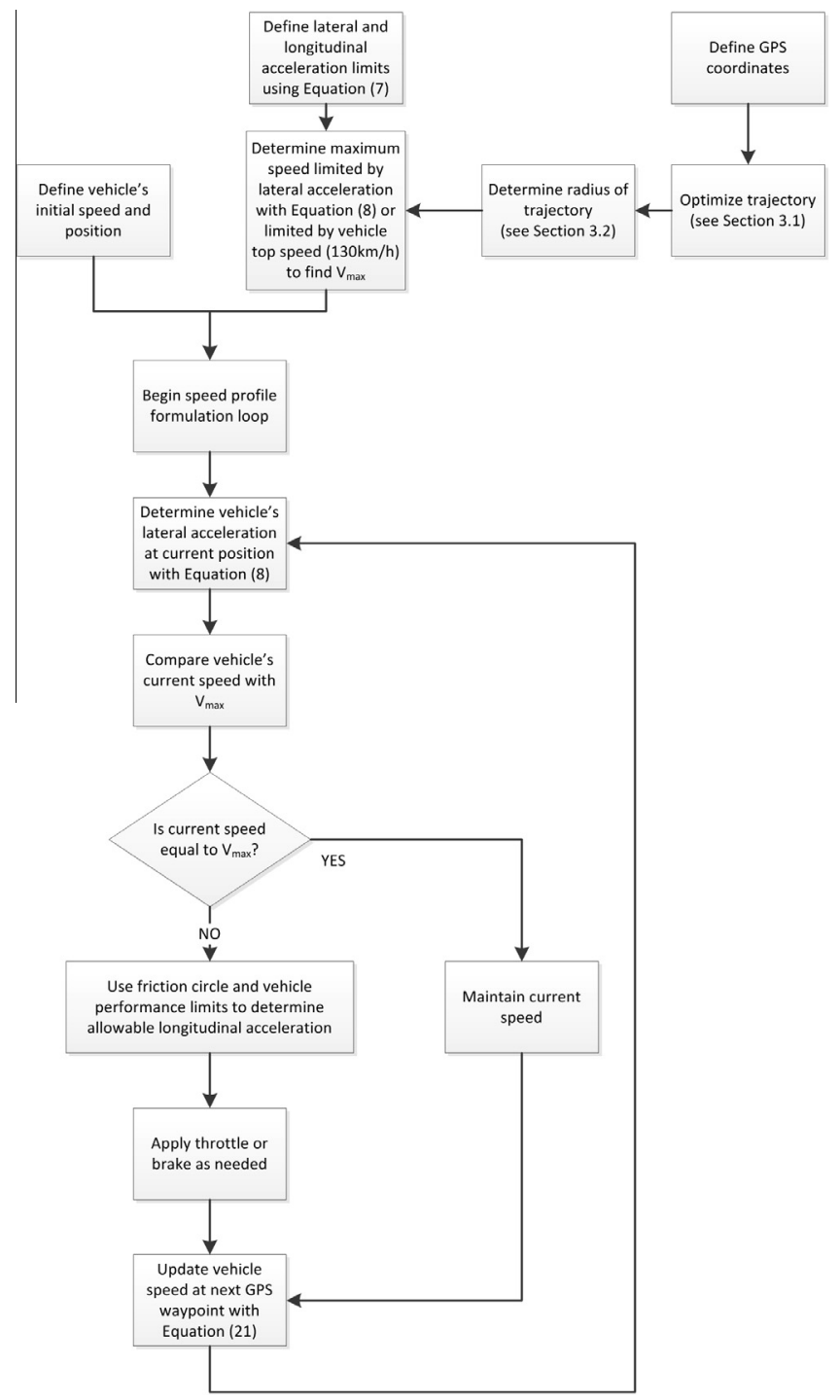

Fig. 11. Flowchart describing formulation of speed profile.

The line bisecting the chord from the two adjacent points is perpendicular to the chord, hence the product of the slope of the chord "line" and the slope of the "bisector" line must be negative one. The gradient of the chord is thus determined and used to determine the gradient of the bisector:

$m_{i}=\frac{\Delta y_{i}}{\Delta x_{i}}=\frac{y_{i+1}-y_{i}}{x_{i+1}-x_{i}}$

The bisector's gradient is given by Eq. (14):

$m_{i}^{\prime}=\frac{-1}{m_{i}}$

Finally the constant term describing the straight line equation of the bisector is determined. Since it is known that the bisector passes through the point $\left(x_{q, i} ; y_{q, i}\right)$, the constant term is calculated with:

$c_{i}=y_{q, i}-m_{i}^{\prime} x_{q, i}$

The centre point of the arc is then at the intersection of the two bisectors, given by Eqs. (16) and (17):

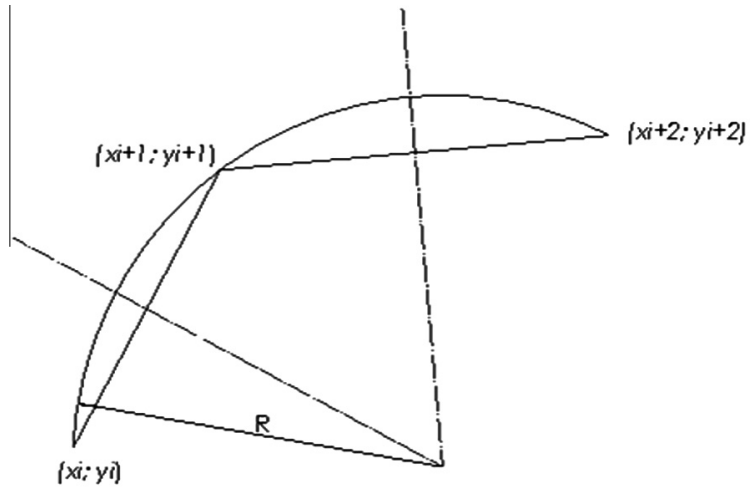

Fig. 12. Constructing an arc through three points.

$x_{R, i}=\frac{c_{i+1}-c_{i}}{m_{i}^{\prime}-m_{i+1}^{\prime}}$

$y_{R, i}=m_{i}^{\prime} x_{R, i}+c_{i}$

The radius of the arc (and hence the radius of curvature) is then determined with Pythagoras' theorem:

$R_{i}=\sqrt{\left(x_{R, i}-x_{i}\right)^{2}+\left(y_{R, i}-y_{i}\right)^{2}}$

Fig. 13(a) shows a spiral with a radius increasing from zero to $50 \mathrm{~m}$. The radius of curvature is confirmed with the outlined algorithm. The radius as a function of arc length is also shown in Fig. 13(b). A slight discrepancy between the true and calculated radius may be seen in Fig. 13(b). This discrepancy is present right at the beginning of the spiral. A possible explanation is that the algorithm cannot calculate a radius of zero. The algorithm works well for radii greater than $5 \mathrm{~m}$ and is thus more than adequate for vehicle trajectory, since a vehicle's turning radius is seldom less than $5 \mathrm{~m}$.

This algorithm may now be used to determine the maximum permissible speed for the vehicle around any track. By specifying a maximum lateral acceleration, the corresponding speed at which this acceleration will occur may be calculated for each point on the trajectory. However, from Eq. (8) it may be seen that when the radius of the trajectory is large, the permissible speed is very high (in a straight line, there is no lateral acceleration) and hence a speed limit of $130 \mathrm{~km} / \mathrm{h}$ was imposed. This is approximately the maximum speed the Land Rover can achieve. Fig. 14 shows the speed as limited by lateral acceleration for the Land Rover around the Gerotek ride and handling track [6]. A maximum lateral acceleration of $0.5 \mathrm{~g}$ was specified in this case.

\subsubsection{Speed limit due to friction circle and vehicle performance}

When accelerating a vehicle both longitudinally and laterally simultaneously, one has to consider the friction circle. Since forces are being generated in two directions in a highly nonlinear system, one cannot simply consider each load condition separately. In Section 3.2.1 the speed 

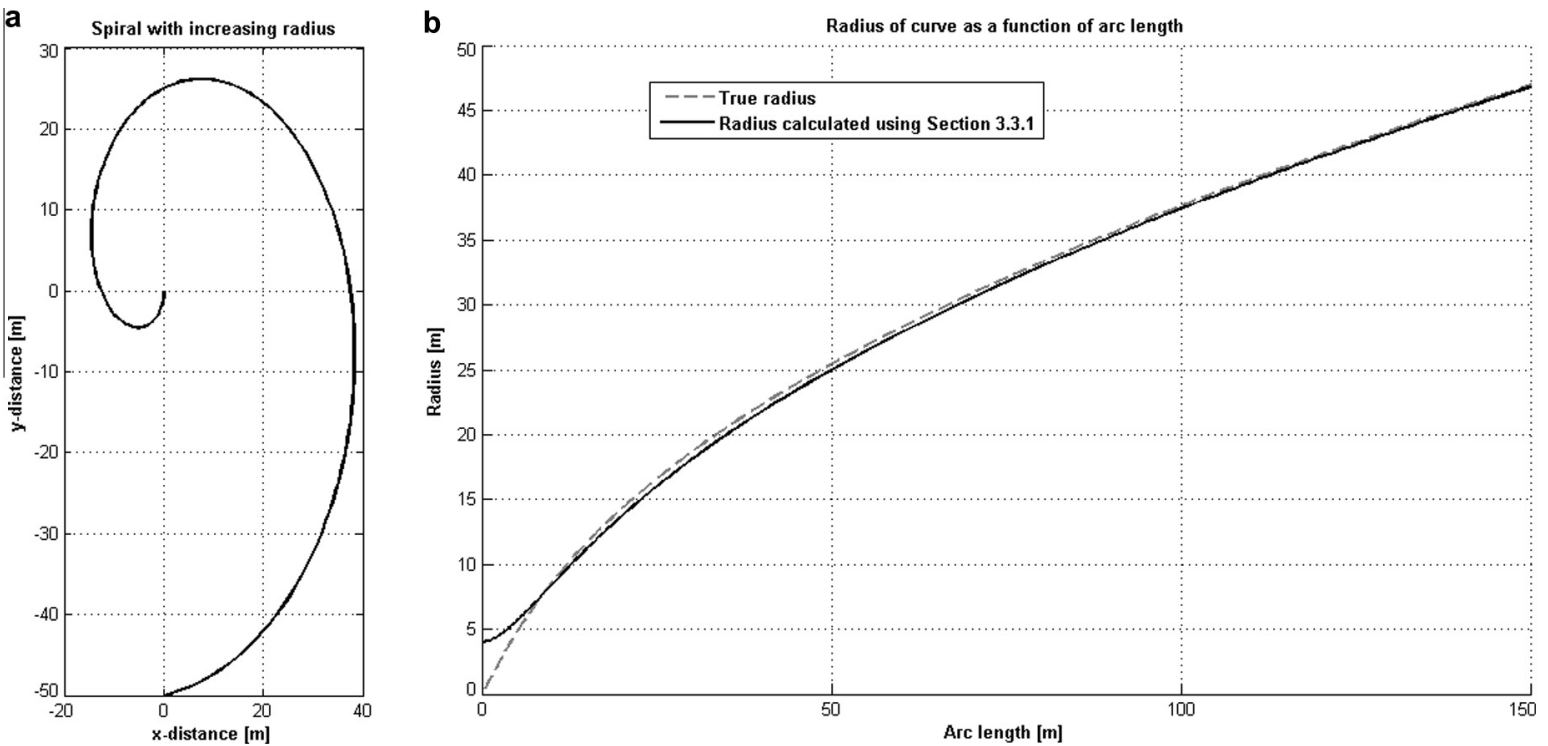

Fig. 13. Spiral with radius increasing from zero to $50 \mathrm{~m}$ and radius of curve as a function of arc length.

profile around the track was optimised for maximum lateral acceleration and hence maximum lateral force generation. The available friction thus limits the vehicle's longitudinal performance. If the friction force is exceeded, the wheels would slip (spin or lock), which may result in an unstable and unsafe situation.

The friction available for longitudinal acceleration is thus governed by the friction circle. The friction force available for longitudinal acceleration is the vector subtraction of the developed lateral force from the friction force limit. This is determined with Eq. (19) [3]:

$A_{x, i}=A_{x, \max } \sqrt{1-\left(A_{y, i} / A_{y, \max }\right)^{2}}$

By imposing a speed limit on the vehicle when negotiating the track, the maximum longitudinal acceleration allowable may be determined. Considering the vehicle's limitations in terms of performance and braking, one may determine the distance required to accelerate and decelerate the vehicle. The vehicle's performance capabilities on a level road are shown as functions of vehicle speed in Fig. 15. Fig. 15(a) shows the maximum acceleration through the gears, Fig. 15(b) indicating the maximum braking performance. These plots were determined from the experimental data, discussed in detail in Section 2.

\subsubsection{Speed profile algorithm}

The speed profile that will be used as the reference speed to be maintained by the vehicle while negotiating the prescribed path is now determined by defining the maximum lateral and longitudinal acceleration deemed safe. A preview distance is defined as a function of the current vehicle speed and the maximum allowable longitudinal acceleration; the function is given in Eq. (20).

$d_{\text {prev }}=V \tau-0.5 A_{\text {maxlong }} \tau^{2}+$ const
The maximum allowable safe speed as limited by the lateral acceleration of the vehicle (see Eq. (8)) for the path to be followed from the current position to the preview point is compared with the current speed of the vehicle. If the allowable speed is higher than the current vehicle speed, the vehicle is allowed to accelerate. The acceleration allowed is determined by taking the lesser of the accelerations due to the vehicle's performance capability (see Fig. 15(a)) and the friction available at the current position (see Eq. (19)). The vehicle's speed at the next position in the path is then updated using the equations of motion assuming constant acceleration (see Eq. (21)):

$V_{i+1}=\sqrt{V_{i}^{2}+2 A_{x} \Delta s_{i}}$

Fig. 16 shows the reference speed for Gerotek's ride and handling track [6]. The solid line is the speed limit imposed by Eq. (8) but with the vehicle's top speed of $130 \mathrm{~km} / \mathrm{h}$ imposed. The dashed line is the reference speed calculated with Eq. (21). It may be noted that the dashed line is never higher than the solid line, indicating that, if the control system tracks the reference speed accurately, the vehicle will never exceed its lateral limits due to excessive speed. This is validated in Section 3.3.

\subsection{ADAMS validation of speed profile}

Before simulation could be performed, some basic control strategies had to be developed to control the actuator positions. These control strategies control the throttle pedal position, the brake line hydraulic pressure and the gear selection that are used as input to the longitudinal speed controller as indicated in Fig. 7. During testing on the vehicle throttle pedal position, steering and gear selection will be performed by the person driving the vehicle, while brake line pressure will be controlled by actuating a pneumatic cylinder mounted to the brake pedal. 


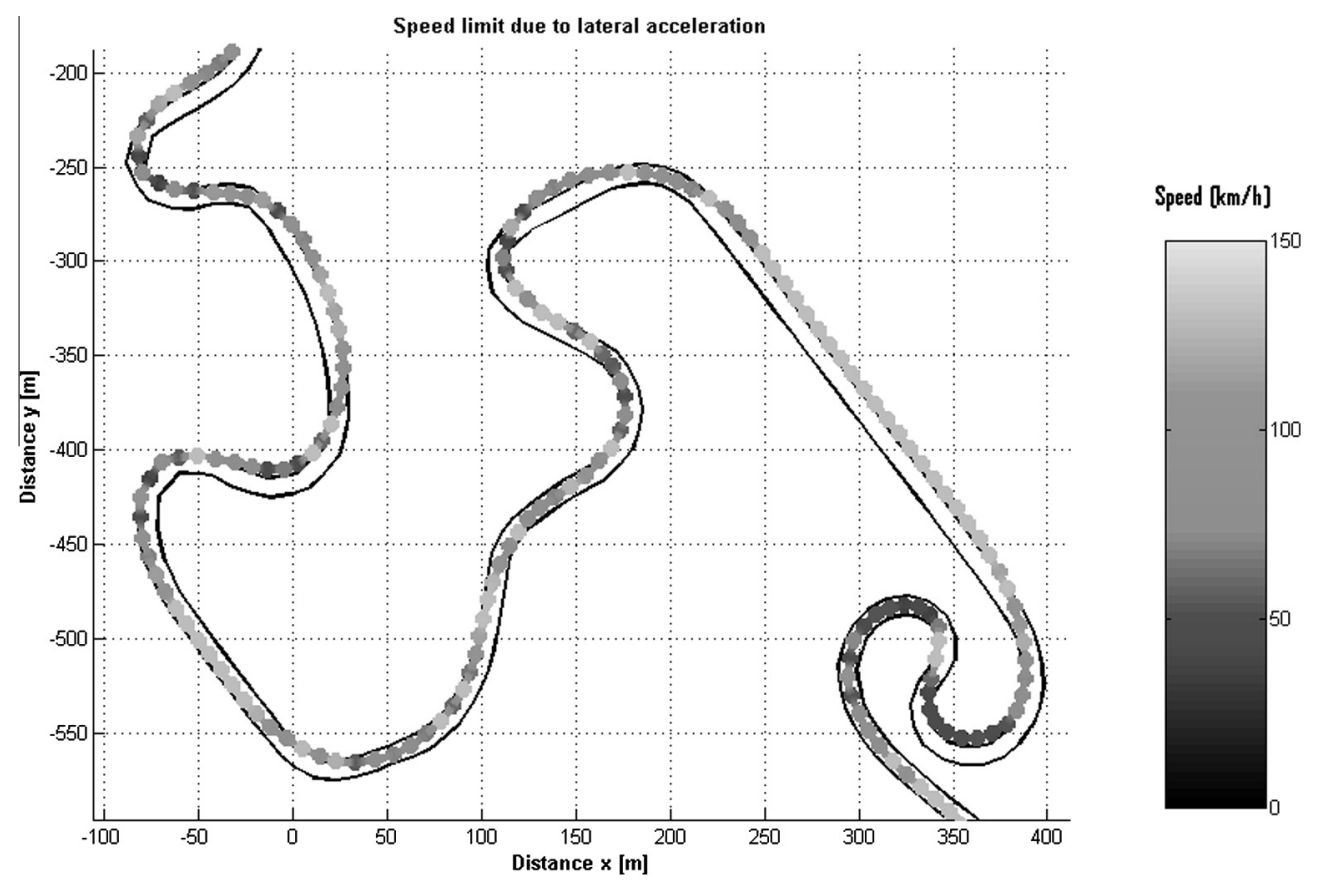

Fig. 14. Enlarged view of speed limit due to lateral acceleration $30 \mathrm{~km} \mathrm{~h}$.

a
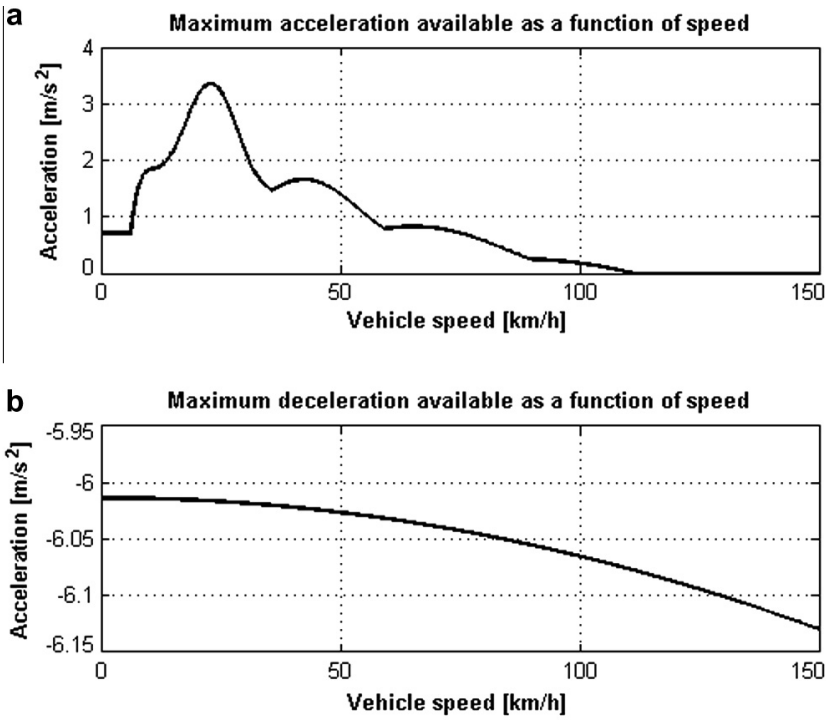

Fig. 15. Land Rover performance limits.

The vehicle acceleration was controlled by controlling the throttle pedal position and brake line hydraulic pressure. The throttle pedal position was controlled with a PID controller and the brake line hydraulic pressure with a PI controller. The gains for these controllers were determined on a trial-and-error basis. The details for of these controllers are not the focus of this paper. The dynamics of the controllers and the actuators are an order of magnitude faster than the vehicle response and the controller gains have a small influence on the vehicle performance.

The velocity error is determined with Eq. (22), with the control system distinguishing between a positive and a negative error to determine whether acceleration or deceleration of the vehicle is necessary. When the error causes the vehicle to brake, the throttle position is immediately set to zero and vice versa. The control signals sent to the vehicle are the throttle pedal position and brake line hydraulic pressure.

$e=V_{\text {ref }}-V$

The gear selection is simply done by monitoring the engine speed. If the engine speed is higher than 3500 RPM the gear is incremented once (unless the vehicle is in top gear) and if the engine speed is less than 1200 RPM the gear is decremented once (unless the vehicle is in first gear). Although this is not an optimum gear shifting regime (the ideal would be to change gears at the intersection of the supply curves in the various gears), this method of controlling the gear selection was chosen due to its simplicity and the ease with which such a feedback control system could be implemented in simulation. On the actual test vehicle the gear selection was done by the driver.

The next step was to simulate the MSC.ADAMS/View model of the Land Rover's response to the inputs generated by the developed control system (which tracks the reference speed profile). The result for negotiating Gerotek Test Facilities' [6] Ride and Handling Track is shown in Fig. 17. It was assumed throughout that the track was flat. Fig. 17(a) shows a plan view of the track. Fig. 17(c) indicates the following: (i) the speed limited by Eq. (8) (solid black line), (ii) the reference speed calculated from Eq. (21) (grey line) and (iii) the speed obtained by the vehicle as the MSC.ADAMS model tries to accelerate and brake the vehicle in order to follow the reference speed. 


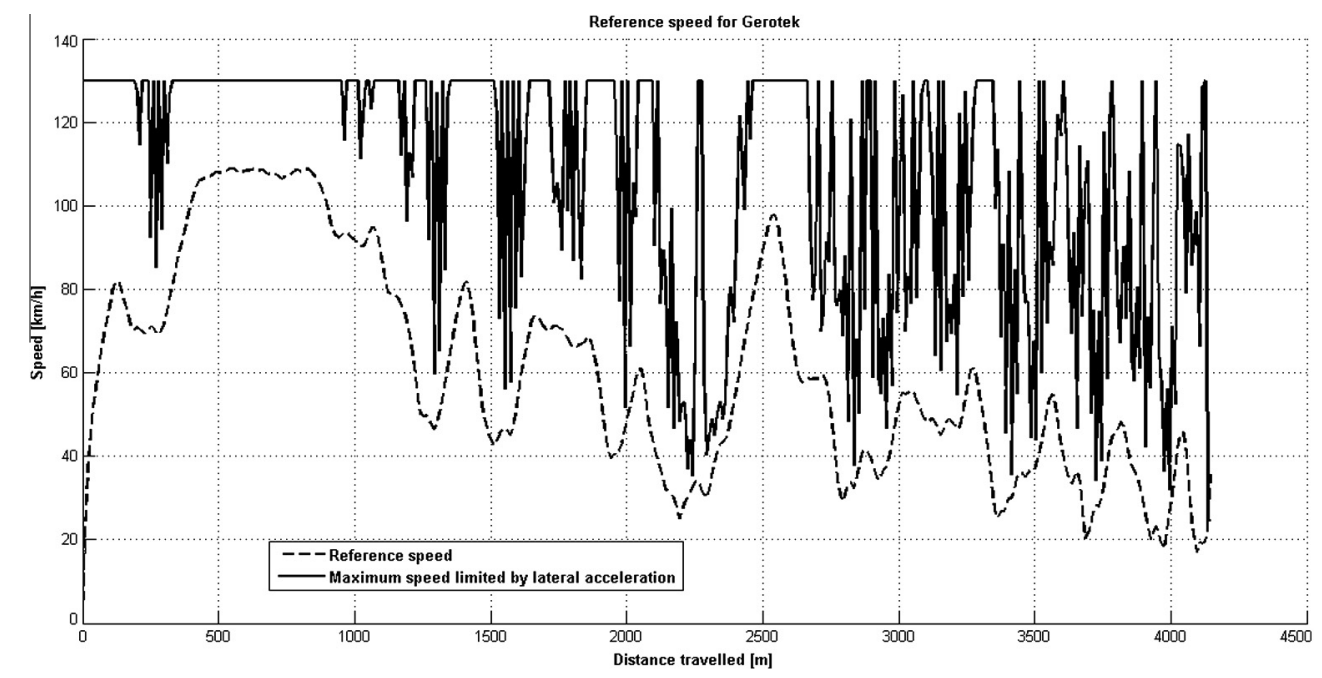

Fig. 16. Reference speed for Geroteks ride and handling track.
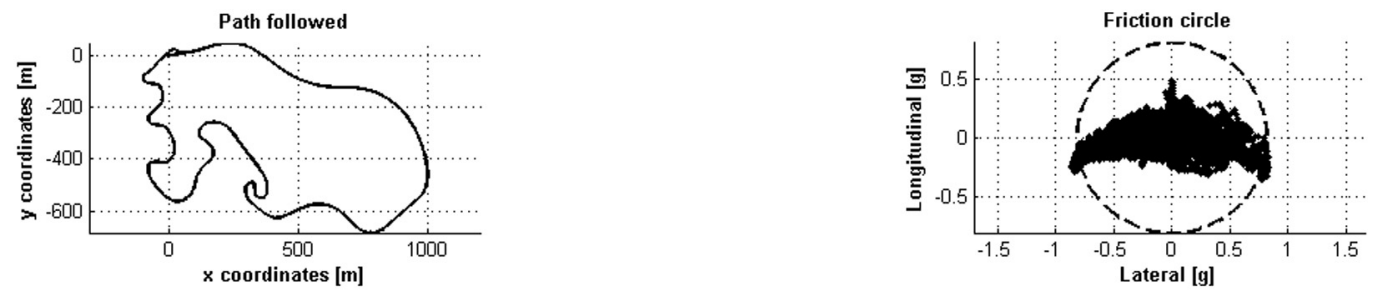

Speed as a function of distance travelled
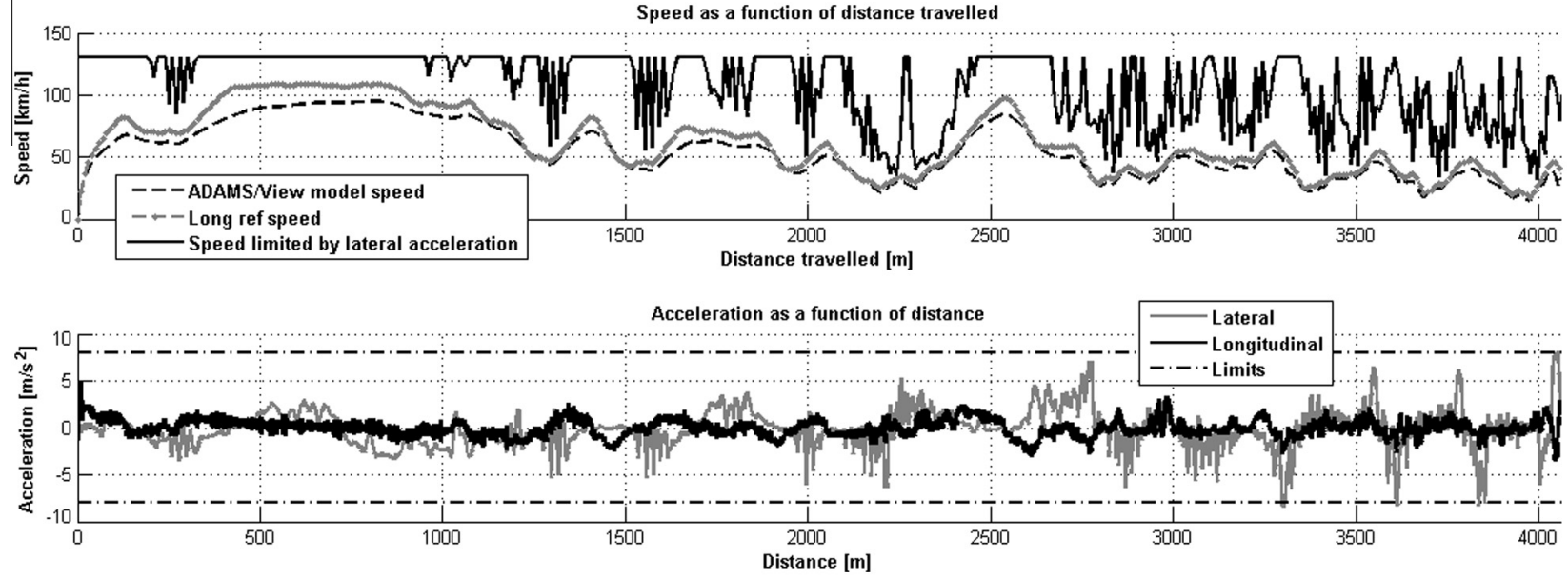

Fig. 17. Simulation results for Geroteks ride and handling track (maximum lateral and longitudinal acceleration of $8 \mathrm{~m} / \mathrm{s}^{2}$ ).

It may be noted that the simulated speed never exceeds the reference speed but that it tracks the prescribed speed closely. The acceleration of the vehicle is slower and therefore cannot follow the prescribed speed. The vehicle does brake fast enough so as to keep the vehicle speed at or below the reference speed. The vehicle is thus underpowered and could drive the track faster with a more powerful engine. The resultant longitudinal and lateral accelerations are plotted in the Fig. 17(d) and the g-g diagram (Fig. 17(b)). The g-g diagram in (Fig. 17(b)) indicates that the vehicle largely stayed within the prescribed acceleration limits (the dashed circle is the friction circle with longitudinal and lateral acceleration limits of $8 \mathrm{~m} / \mathrm{s}^{2}$ ). The driver model used during the simulations maintained control over the vehicle at all times, even though the prescribed limits are very close to the vehicle's limits. The simulations were repeated for several race tracks of which GPS coordinates are available.

Simulation results indicate that the vehicle performs as planned, tracking the reference speed profile closely (considering the slow dynamics of a vehicle such as a Land Rover Defender) and that the accelerations measured largely stay within the friction circle. Hence the development of the longitudinal control system was deemed to have been successful. The next step in the process is experimental validation of the longitudinal control system. 


\section{Experimental validation}

The measurement transducers used during the experimental validation were:

- An accelerometer at the centre of gravity $(\mathrm{CG})$ of the test vehicle to measure longitudinal and lateral acceleration.

- A VBox III Differential GPS [12] to measure the vehicle's speed and GPS position.

- A hydraulic pressure transducer to measure the brake line hydraulic pressure.

- A rotary potentiometer to measure the throttle position.

A PC/104 form factor embedded computer with a Diamond MM-AT-12-bit Analogue to Digital I/O Module [13] was used for data acquisition. Since the longitudinal control is a very slow dynamic process, a sample frequency of $100 \mathrm{~Hz}$ was deemed sufficient for both data acquisition and control.

The VBox III Differential GPS [12] is a data logging system that can $\log$ GPS data at $100 \mathrm{~Hz}$. A Local Differential GPS Base station was used in conjunction with the VBox III to improve the positional accuracy to within $100 \mathrm{~mm}$. The same system was used by Botha [5] as positional input to his steering controller with resounding success.

A laptop, connected to the PC/104 computer via a TCP network, was used in conjunction with the PC/104 to obtain the GPS coordinates and vehicle speed from the VBox III. The measured velocity and prescribed velocity were compared and a velocity error calculated. The velocity error was used to determine whether the brakes had to be applied or not. The PC/104 computer was used to control the brake actuator, generating an analogue output to control the brake line pressure and the driver controlled the throttle, clutch, gear lever and steering. A corresponding prompt

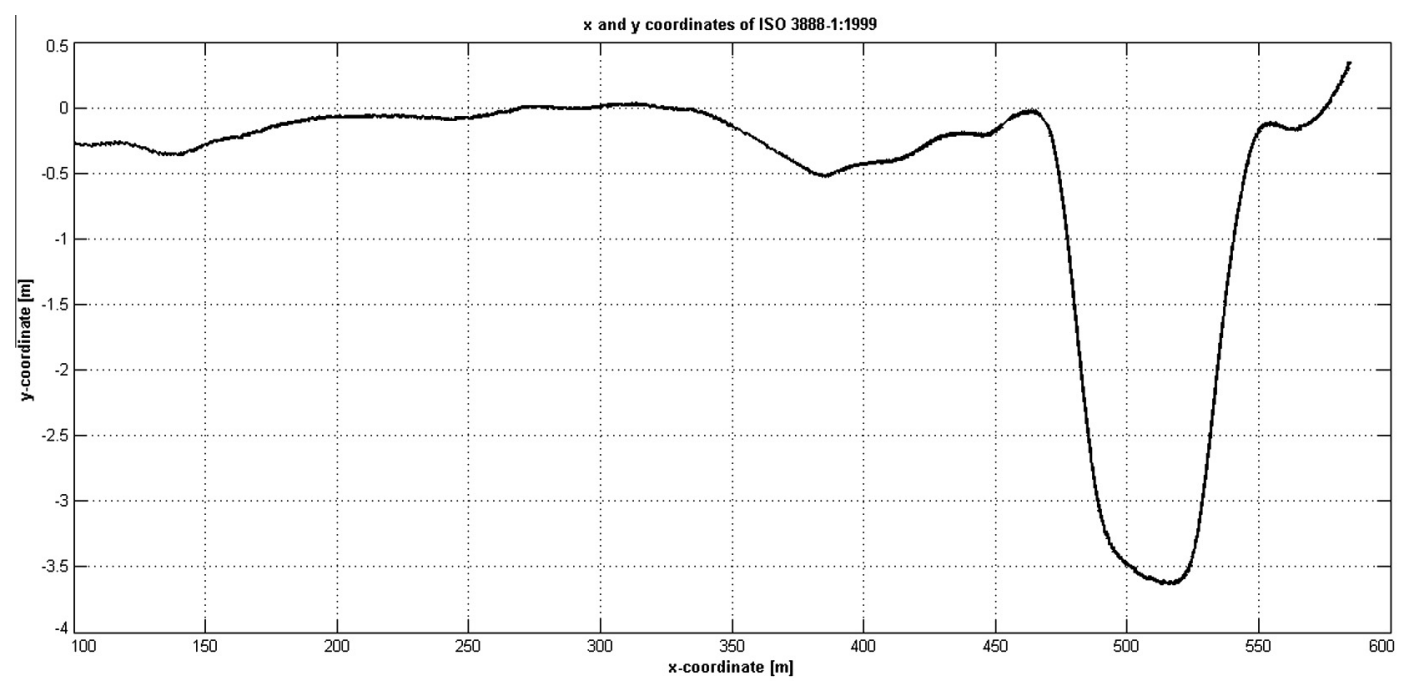

Fig. 18. Recorded $x$ and $y$ coordinates of ISO 3888-11999 severe...change.

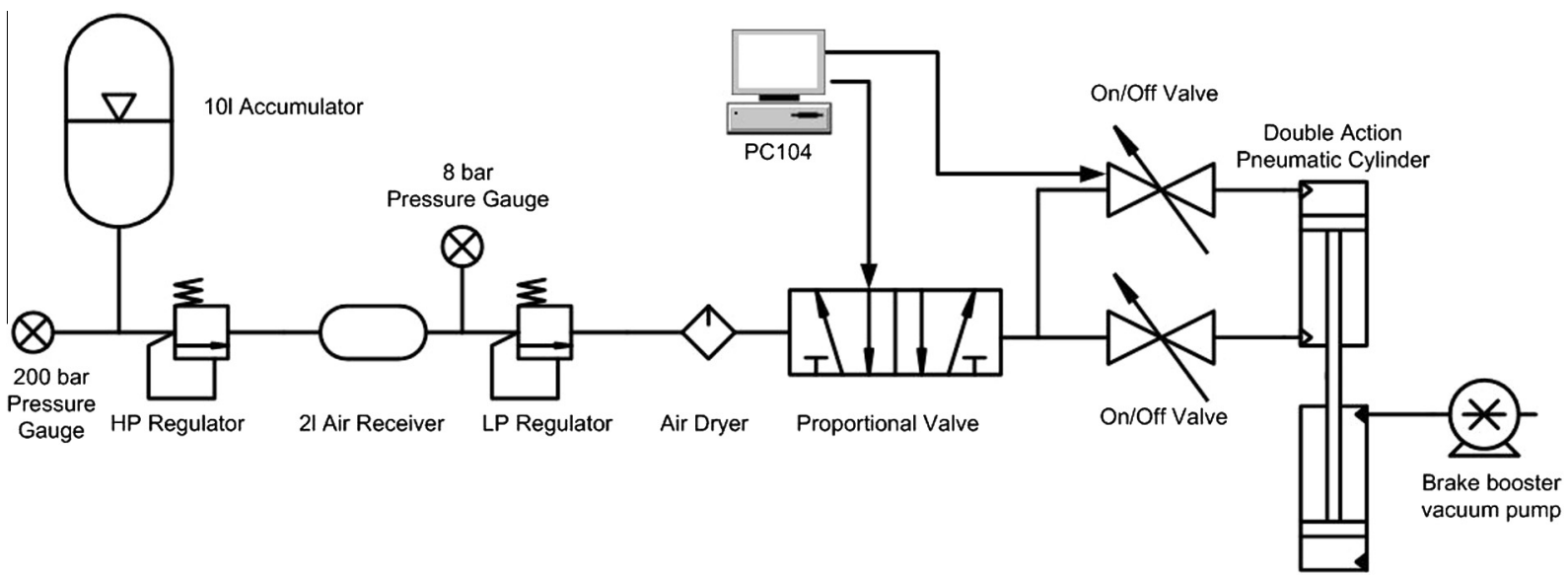

Brake master cylinder

Fig. 19. Schematic layout of experimental setup. 
was given to the driver as an indication whether the brakes were going to be applied or not so that the driver could release the throttle and depress the clutch.

The only actuator used for experimental validation was the pneumatic actuator that controls the vehicle's deceleration by braking. The decision to only make use of the braking control system is that it is more representative of the intended application of the developed driver assist system. The driver manually operated the throttle, clutch, gears and steering during the validation run. While using a human driver rather than the fully autonomous control systems described in Section 1 may cause significant errors with respect to the path following, this gives an indication as to the robustness of the driver assist system. The
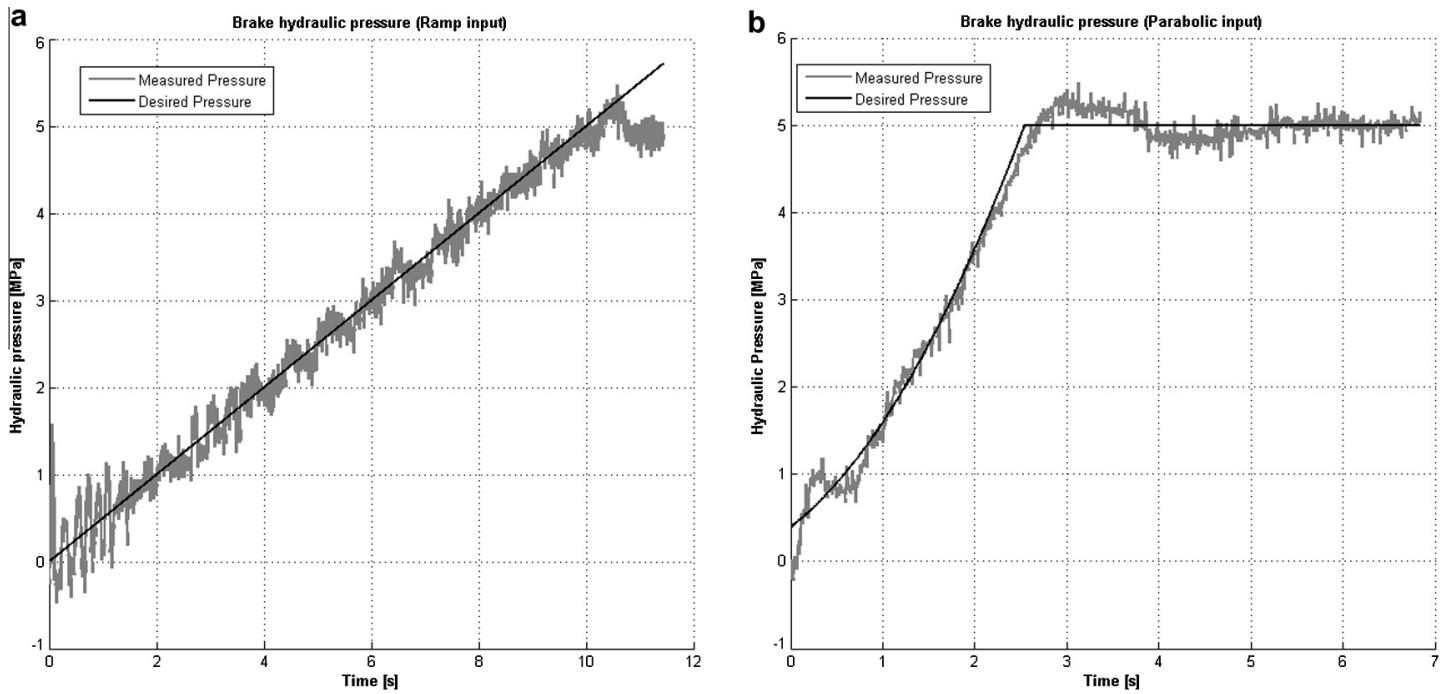

Fig. 20. Brake actuator response to ramp (left) and parabolic (right) input.
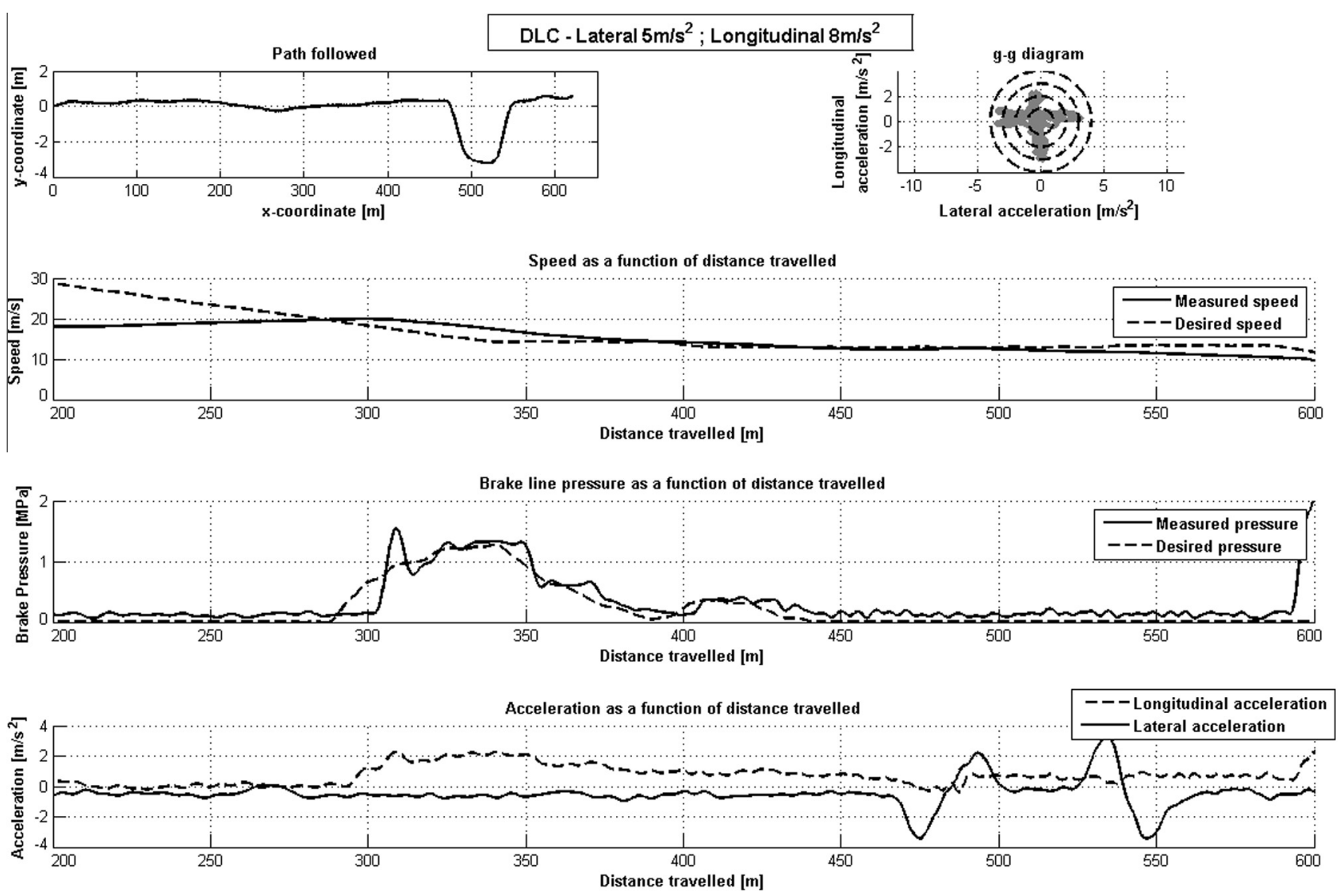

Fig. 21. Double lane change with lateral and longitudinal limits of $5 \mathrm{~m} / \mathrm{s}^{2}$ and $8 \mathrm{~m} / \mathrm{s}^{2}$ respectively. 
application of the brakes to prevent the driver from exceeding the lateral acceleration limits of the vehicle was deemed to be sufficient for validation. During the run a prompt was displayed on a computer screen indicating whether the vehicle's speed was too high or too low. If the brakes were being applied, the driver disengaged the clutch. This was to prevent the vehicle from stalling while performing the double lane change manoeuvre (this may happen if the engine speed drops below idle speed due to the vehicle driving too slowly for the selected gear).

\subsection{Experimental procedure}

For the purpose of experimental validation, a severe double lane change manoeuvre (ISO 3888-1:1999 [14]) was performed. The boundaries of the severe double lane change were laid out with high visibility cones. The experimental procedure may be described as follows:

(1) Record the path to be driven by driving at low speed with the GPS.

(2) Define the maximum allowable lateral and longitudinal acceleration.

(3) Calculate the speed profile, as discussed in Section 3.2 (pre-processing).

(4) Perform the severe double lane change manoeuvre.
The GPS coordinates of the path to be driven are recorded with the VBox III. These GPS coordinates are converted to $x$ and $y$ coordinates (shown in Fig. 18) that are then used to determine the speed profile. The speed profile is determined as a function of GPS position.

While negotiating the severe double lane change, the vehicle compares its current speed (as measured with the VBox III) with the prescribed speed at that position. If the measured speed is below the prescribed speed, the vehicle is allowed to accelerate. The vehicle's brakes are applied if the measured speed is above the prescribed speed.

Fig. 19 shows a schematic description of the brake actuator setup and control system. The actuator system consists of:

a. 200 bar, 101 air accumulator, pressure regulators and a 10 bar, 21 air receiver.

b. Proportional and on-off air valves.

The ability of the brake pressure actuator to supply the correct pressure to the brake system was verified by applying ramp and parabolic requirements. Fig. 20 indicates the prescribed pressures as well as the measured pressures. The brake actuator and control system follows the required pressure with very good accuracy and is deemed suitable for use in autonomous braking of the vehicle.
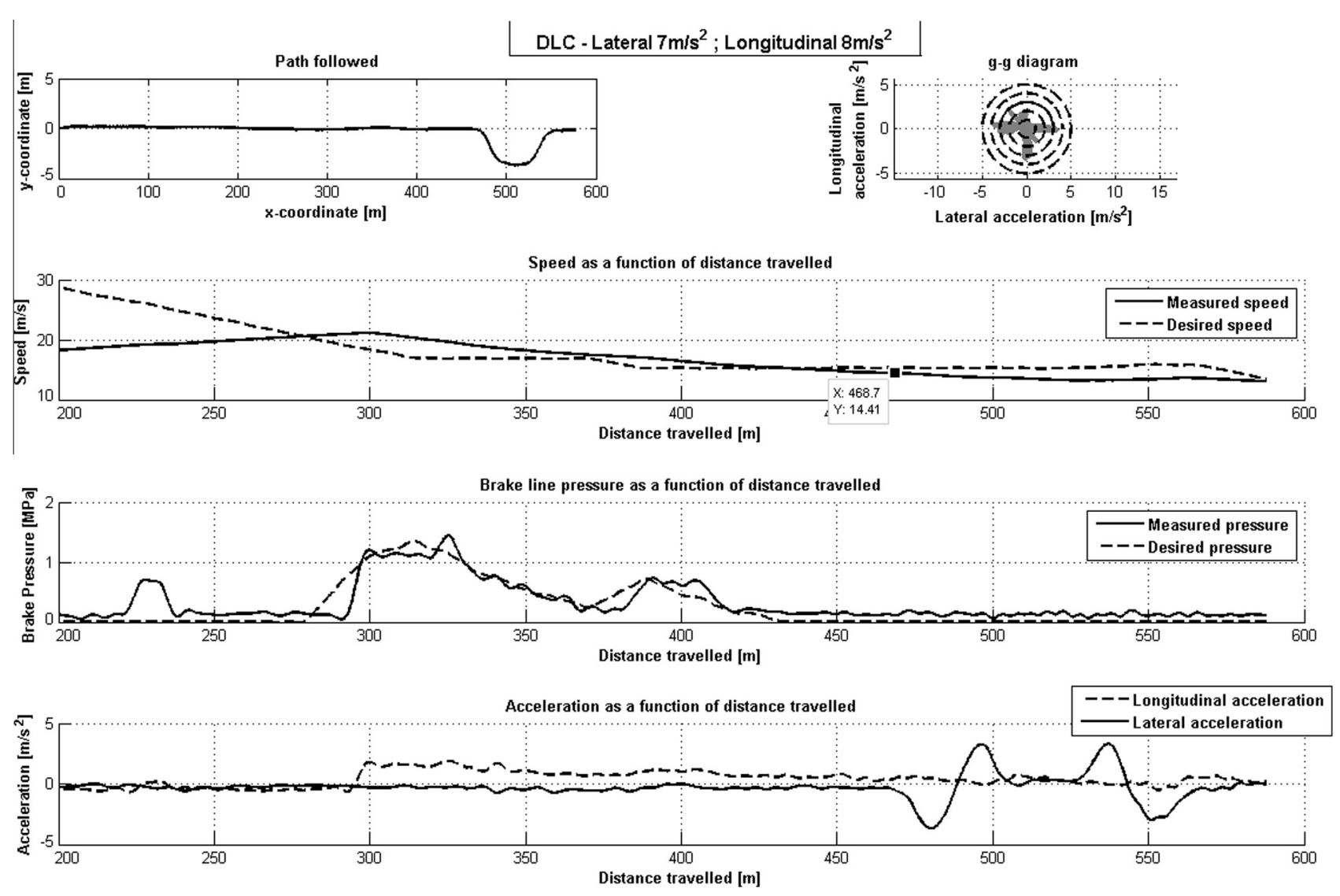

Fig. 22. Double lane change with lateral and longitudinal limits of $7 \mathrm{~m} / \mathrm{s}^{2}$ and $8 \mathrm{~m} / \mathrm{s}^{2}$ respectively. 


\subsection{Experimental results}

The results obtained from the experiments are plotted in Figs. 21-23. Each plot contains five graphs, namely:

a. The $x$ and $y$ coordinates of the path followed during the test run in the top left corner.

b. A g-g diagram showing lateral and longitudinal acceleration during the run. Included in the $\mathrm{g}-\mathrm{g}$ diagram are dashed friction circles for incrementing longitudinal and lateral acceleration, i.e. the innermost circle is for $1 \mathrm{~m} / \mathrm{s}^{2}$ lateral and longitudinal acceleration, the second circle for $2 \mathrm{~m} / \mathrm{s}^{2}$ lateral and longitudinal acceleration and so forth.

c. The graph second from the top is a plot of the measured vehicle speed (solid line) and the prescribed vehicle speed (dashed line).

d. The third graph from the top shows the desired brake line pressure (dashed line) and the measured brake line pressure (solid line).

e. The bottom graph plots lateral (solid line) and longitudinal acceleration (dashed line) as functions of the distance travelled from the starting point.

The double lane change manoeuvre was performed at various prescribed lateral and longitudinal acceleration limits. Initially, the prescribed longitudinal acceleration limit was set to $8 \mathrm{~m} / \mathrm{s}^{2}$ while doing multiple double lane change manoeuvres at various prescribed lateral acceleration limits. The prescribed longitudinal acceleration limit was then reduced to $3 \mathrm{~m} / \mathrm{s}^{2}$, a value more readily associated with everyday driving.

\subsubsection{DLC at $5 \mathrm{~m} / \mathrm{s}^{2}$ lateral and $8 \mathrm{~m} / \mathrm{s}^{2}$ longitudinal acceleration}

The results of the double lane change manoeuvre with prescribed acceleration limits of $5 \mathrm{~m} / \mathrm{s}^{2}$ lateral and $8 \mathrm{~m} / \mathrm{s}^{2}$ longitudinal acceleration are shown in Fig. 21. A vehicle speed of approximately $13 \mathrm{~m} / \mathrm{s}$ was maintained during the double lane change manoeuvre, resulting in a maximum lateral acceleration of $3.5 \mathrm{~m} / \mathrm{s}^{2}$. The maximum longitudinal acceleration recorded during the manoeuvre was $3 \mathrm{~m} / \mathrm{s}^{2}$. The control system managed to adequately track the desired brake line pressure and hence the speed during the double lane change was very close to the prescribed speed and the acceleration in both the longitudinal and lateral directions stayed well within the prescribed limits.

\subsubsection{DLC at $7 \mathrm{~m} / \mathrm{s}^{2}$ lateral and $8 \mathrm{~m} / \mathrm{s}^{2}$ longitudinal acceleration}

The highest prescribed lateral acceleration limit tested at was $7 \mathrm{~m} / \mathrm{s}^{2}$ and the results thereof are shown in Fig. 22 .
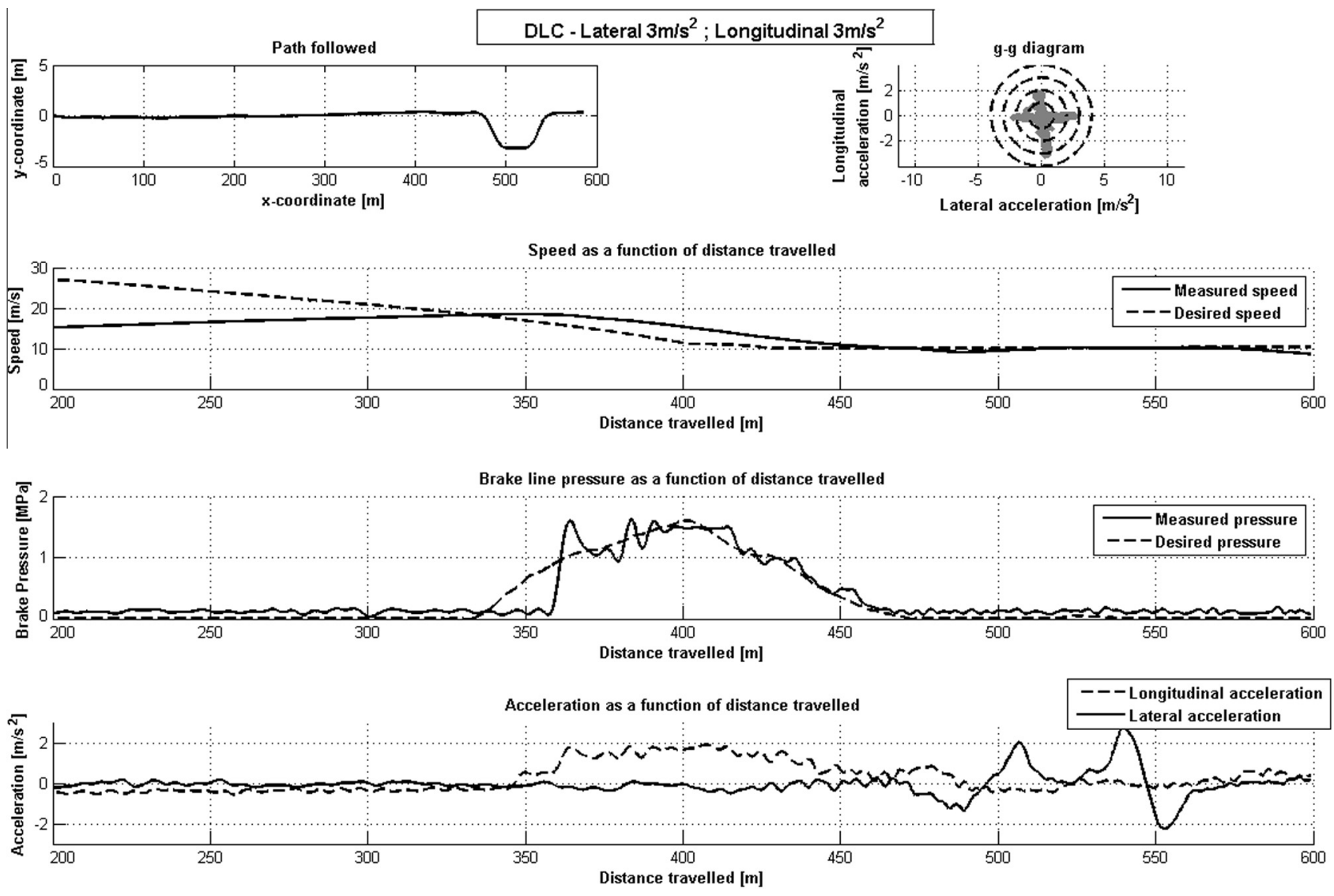

Fig. 23. Double lane change with lateral and longitudinal limits of $3 \mathrm{~m} / \mathrm{s}^{2}$ and $3 \mathrm{~m} / \mathrm{s}^{2}$ respectively. 
The maximum lateral and longitudinal accelerations measured during the double lane change manoeuvre were $4 \mathrm{~m} / \mathrm{s}^{2}$ and $3.2 \mathrm{~m} / \mathrm{s}^{2}$ respectively, well within the prescribed limits. The double lane change was negotiated at $14 \mathrm{~m} / \mathrm{s}$, slightly lower than the speed as prescribed by the speed profile algorithm and hence the lateral acceleration was significantly lower than the prescribed limit. The actuator applying the brakes adequately tracked the desired brake line pressure, but the vehicle lost too much speed. This may be attributed to driver error, the driver neglecting to maintain the speed once the double lane change had been entered.

\subsubsection{DLC at $3 \mathrm{~m} / \mathrm{s}^{2}$ lateral and $3 \mathrm{~m} / \mathrm{s}^{2}$ longitudinal acceleration}

The prescribed longitudinal acceleration limit was reduced to $3 \mathrm{~m} / \mathrm{s}^{2}$, with the lateral acceleration limit at $3 \mathrm{~m} / \mathrm{s}^{2}$ and the experimental results are shown in Fig. 23. The reason for the low prescribed lateral acceleration limit is because the low prescribed longitudinal acceleration limit prevented the Land Rover from reaching a speed high enough to excite high lateral accelerations in the previous tests. During the double lane change, the maximum lateral acceleration measured was $2.8 \mathrm{~m} / \mathrm{s}^{2}$ and the maximum longitudinal acceleration $2 \mathrm{~m} / \mathrm{s}^{2}$, the vehicle thus stayed within the prescribed acceleration limits. The double lane change was negotiated at $10 \mathrm{~m} / \mathrm{s}$, similar to the speed in Section 4.2.1 which has the same prescribed lateral limit.

\subsection{Discussion of results}

During all of the double lane change manoeuvres performed the lateral acceleration of the vehicle was reduced as desired by controlling the longitudinal behaviour of the vehicle by braking. In all of the tests the measured accelerations never exceeded the prescribed limits. The vehicle reduced speed when it exceeded the prescribed speed, resulting in the measured accelerations being within the desired limits.

The pneumatic actuator used to control the vehicle's brakes adequately tracked the desired brake line pressure in all of the tests, even when noise was present. This validated the design of the PID controller used to control the brake line pressure.

The prescribed longitudinal acceleration limit was only reached when the prescribed limit was low. When the prescribed longitudinal acceleration was high, the vehicle braked early, resulting in lower longitudinal accelerations being measured. A possible explanation for this is that the preview distance used to determine when to apply the brakes is too conservative, effectively looking too far ahead when the prescribed longitudinal acceleration is high.

\section{Conclusions}

The rollover risks of a vehicle (SUV) can be considerably reduced by controlling the maximum lateral acceleration that the vehicle can achieve. Lateral acceleration can be limited by limiting the vehicle speed as a function of turn radius. The trajectory of the track or road to be followed was optimised to maximise the radius of the path to be followed. This optimised trajectory was used in conjunction with a longitudinal speed driver model to define a speed limit for the vehicle at each point on the trajectory. The driver model took into account prescribed longitudinal and lateral acceleration limits and the vehicle's lateral acceleration limits, the friction available for combined longitudinal and lateral force generation (due to the friction circle concept) and the vehicle's longitudinal performance capabilities to formulate a safe speed at each point of the path being followed. These limits can be based on friction estimates, lookup tables (in response to an input from the driver telling the vehicle the nature of the terrain or from a terrain response system). The process of optimising the trajectory and determining a safe speed was termed the speed profile algorithm. A longitudinal control system was developed to control vehicle speed. The system aims to reduce vehicle speed early enough to prevent the vehicle from entering a dangerous situation, i.e. it acts before electronic stability control (ESC) and similar driver assist systems need to start intervening.

The performance of the longitudinal control system was evaluated in simulation using the validated MSC.ADAMS/View model of the Land Rover Defender. Several racetracks of which the GPS coordinates are available were used for simulation purposes. A speed profile was developed for each racetrack and MSC.ADAMS/View was used in conjunction with MATLAB and Simulink to simulate the Land Rover Defender negotiating these tracks. In all of the simulations, the steering controller maintained control over the vehicle, even though the model was operating very close to the vehicle's limits. Throughout the simulations, the lateral and longitudinal accelerations stayed within the prescribed limits. The simulation results indicated that the longitudinal control system was safe for experimental validation on the Land Rover Defender.

Experimental validation of the longitudinal control system was done by performing an ISO 3888-1:1999 severe double lane change manoeuvre. Speed profiles for the double lane change were formulated with various prescribed longitudinal and lateral acceleration limits. The pneumatic actuator used for controlling the brake line pressure was used to prevent the vehicle from exceeding the speed deemed safe by the speed profile algorithm. During all the test runs, the vehicle's measured lateral and longitudinal accelerations were within the prescribed limits. The system was found to be conservative, rarely reaching the prescribed longitudinal acceleration. The advantage of the system being conservative is that it adds robustness to the speed profile algorithm, allowing it to be used for any prescribed acceleration limits. 


\section{Recommendations}

Multiple aspects which could improve the longitudinal control system and speed profile algorithm at higher prescribed acceleration limits have been identified. These include:

- The development of software that can optimise the trajectory in real time, taking account of the vehicle's current position, may result in a safer speed limit being formulated.

- Additional sensors that may detect and can hence be used to avoid obstacles may be integrated with the system, e.g. lane departure warning systems. This may result in a more fully autonomous vehicle.

- By incorporating all the driver aids developed on the Land Rover Defender, significant improvements in vehicle safety may be achieved. A strategy to decide when to employ which driver aid will need to be developed.

- Using parameter estimation techniques to estimate the friction coefficient between the tyres and the terrain one may implement this control system on almost any terrain and further improve the occupants' safety.

Performing these recommendations may result in significant improvements in the vehicle's performance, safety and stability.

\section{References}

[1] Thrun S. Winning the darpa grand challenge. In: Knowledge discovery in databases: PKDD 2006. Berlin, Heidelberg: Springer; 2006. p. 4-4.
[2] Urmson C, Anhalt J, Bagnell D, Baker C, Bittner R, Clark MN. Autonomous driving in urban environments: Boss and the urban challenge. J Field Robot 2008;25(8):425-66.

[3] Braghin F, Melzi S, Sabbioni E, Poerio N. Identification of the optimal trajectory for a race driver. In: Proceedings of the ASME 2011 international design engineering technical conferences \& computers and information in engineering conference, 28-31 August 2011. American Society of Mechanical Engineers, Washington, DC, USA; 2011.

[4] Uys PE, Els PS, Thoresson MJ. Suspension settings for optimal ride comfort of off-road vehicles travelling on roads with different roughness and speeds. J Terramech 2007;44:163-75.

[5] Botha TR. High speed autonomous off-road vehicle steering. MEng(Mechanical) thesis, University of Pretoria; 2011. <http:// www.upetd.up.ac.za/thesis/available/etd-11212011-125411/>.

[6] Gerotek test facilities; 2013. <http://www.armscordi.com/SubSites/ Gerotek1/Gerotek01_landing.asp $>$ [accessed 12.03.13].

[7] Gillespie TD. Fundamentals of vehicle dynamics. Warrendale, PA: Society of Automotive Engineers; 1992.

[8] Botha TR. Brake control - autonomous vehicle brake system. Unpublished BEng(Mechanical) final year project, University of Pretoria; 2008.

[9] Garmin. What is GPS?; 2013. <http://www8.garmin.com/aboutGPS/ $>$ [accessed 18.07.13].

[10] Dork RA. Satellite navigation systems for land vehicles. IEEE Aerospace Electr Syst Mag 1987;2(5):2-5.

[11] Batavia PH, Pomerlau DA, Thorpe CE. Predicting lane position for roadway departure prevention. In: Proceedings of the IEEE intelligent vehicles symposium; 1998

[12] Racelogic. VBOXTools software manual ver1.9; 2008. <http:// www.racelogic.co.uk/_downloads/vbox/Manuals/Data_Loggers/ RLVB3_Manual\%20-\%20English.pdf> [accessed 12.03.13].

[13] Diamond Systems Corporation. DIAMOND-MM-AT user manual V1.21. Diamond Systems Corporation, Newark, CA; 2004.

[14] International Organization for Standardization. ISO 3888-1:1999: passenger cars - test track for a severe lane-change manoeuvre - Part 1: Double lane-change. Geneva, ISO; 1999. 